Journal of Nuclear Medicine, published on June 24, 2021 as doi:10.2967/jnumed.121.262279

\title{
A longitudinal PET/MR imaging study of colony stimulating factor-1 receptor-mediated microglia depletion in experimental stroke
}

Cristina Barca ${ }^{1,2}$, Amanda J Kiliaan ${ }^{3}$, Claudia Foray ${ }^{1,2}$, Lydia Wachsmuth ${ }^{4}$, Sven Hermann ${ }^{1}$, Cornelius Faber ${ }^{4}$, Michael Schäfers ${ }^{1,5}$, Maximilian Wiesmann ${ }^{3}$, Andreas H. Jacobs ${ }^{1,2,6,{ }^{*}}$ and Bastian Zinnhardt ${ }^{1,2,5,7 \#, *}$

${ }^{1}$ European Institute for Molecular Imaging (EIMI), University of Münster, Germany

${ }^{2}$ PET Imaging in Drug Design and Development (PET3D)

${ }^{3}$ Department of Medical Imaging, Anatomy, Radboud University Medical Center, The Netherlands

${ }^{4}$ Clinic of Radiology, Translational Research Imaging Center (TRIC), University Hospital Münster, Germany

${ }^{5}$ Department of Nuclear Medicine, University Hospital Münster, Germany

${ }^{6}$ Department of Geriatrics and Neurology, Johanniter Hospital, Germany

7\#Biomarkers \& Translational Technologies (BTT), Pharma Research \& Early Development (pRED),

F. Hoffmann-La Roche Ltd., Switzerland

${ }^{*}$ Equal contribution 
Corresponding authors: Andreas $\mathrm{H}$. Jacobs (PI) and Cristina Barca (PhD)

European Institute for Molecular Imaging (EIMI)

University of Münster, Waldeyerstrasse 15

D-48149 Münster

e-mail addresses:

ahjacobs@uni-muenster.de

cristina.barca@uni-muenster.de

Words: 5018

Short title: CSF-1R inhibition in stroke

Immediate Open Access: Creative Commons Attribution 4.0 International License (CC BY) allows users to share and adapt with attribution, excluding materials credited to previous publications. License: https://creativecommons.org/licenses/by/4.0/. Details: https://jnm.snmjournals.org/page/permissions.

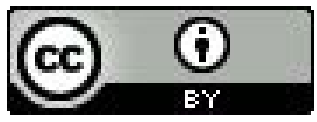




\section{ABSTRACT}

Microglia-induced neuroinflammation after stroke contributes to the exacerbation of postischemic damage but also supports neurorestorative events. Longitudinal molecular imaging of microglia-targeted therapies will support the assessment of target engagement, therapy efficacy, and deciphering the mode of action. We investigated the effects of chronic colony stimulating factor-1 receptor (CSF-1R) inhibitor-mediated microglia depletion on translocator protein (TSPO)dependent neuroinflammation and cerebrovascular parameters using positron emission tomography $(\mathrm{PET}) /$ magnetic resonance $(\mathrm{MR})$ imaging. Methods: $\mathrm{N}=40 \mathrm{C} 57 \mathrm{BL} / 6$ mice underwent a 30 minutes transient middle cerebral artery occlusion (tMCAo) and were randomly assigned to either control group or treated with CSF-1R inhibitor (PLX5622; Plexxikon Inc.). $\mathrm{N}=8$ mice/group were used for 18F-DPA-714 (TSPO) PET imaging at days 7, 14, 21, and 30 post ischemia and behavioural tests prior and after surgery. An extra group of $n=8$ mice/group underwent MR imaging including $\mathrm{T}_{2}$-weighted (infarct), perfusion- (cerebral blood flow) and diffusion-weighted (water diffusion, cellular density) at days 1, 3, 7, 14, 21 and 30. Ex vivo analysis (immunoreactivity, gene expression) were performed to characterize the inflammatory environment. Results: We demonstrated that long-term CSF-1R inhibition transiently decreases the TSPO-PET signal within the infarct. Residual TSPO activity was partly due to potentially resistant Iba- $1^{+}$cell populations with low CSF-1R and transmembrane 119 (TMEM119) expression. The decrease in selected proand anti-inflammatory marker expression suggested an apparent global dampening of the neuroinflammatory response. Furthermore, the temporal changes of the MRI parameters highlighted treatment-induced effects on reperfusion and tissue homeostasis, associated with impaired motor functions at late stages. Conclusion: Longitudinal TSPO-PET/MR imaging allows 
the assessment of target engagement and optimization of drug efficiency. PLX5622 has promising immunomodulatory effects and the optimal therapeutic time window for its application needs to be defined.

\section{KEYWORDS}

Colony stimulating factor-1 receptor, microglia, stroke, 18F-DPA-714, MRI 


\section{INTRODUCTION}

Neuroinflammation and microglial activity are key contributors to stroke pathogenesis (1). Microglia are essential in brain immunosurveillance, monitoring homeostasis and shaping the synaptic landscape at steady-state. In pathological conditions such as cerebral ischemia, they undergo transcriptomic alterations resulting in an early protective anti-inflammatory microglia within the first week, quickly switching to a detrimental pro-inflammatory phenotype around days 10-14 post ischemia, worsening inflammation and tissue damage (2). Therefore, modulating microglia reactivity may offer a new therapeutic approach in the treatment of cerebral ischemia.

The colony stimulating factor-1 receptor (CSF-1R) is a well-described tyrosine kinase involved in differentiation, proliferation and survival of resident microglial cells but also perivascular and peripheral macrophages (monocytes) and other cell types (osteoclasts, dendritic cells) (3). Recently, the selective CSF-1R inhibitor PLX5622 was reported as an efficient treatment to deplete microglial cells in healthy $(4,5)$ and pathological conditions $(6,7)$. In a healthy brain, CSF-1R inhibition leads to $50 \%$ depletion after three days and near-complete depletion of Iba- $1^{+}$ microglial cells within seven days with few side effects. In inflammatory environment, PLX5622 treatment led to beneficial outcomes: it efficiently depleted microglial cells, shifted the remaining cells towards a more anti-inflammatory phenotype and alleviated/improved symptoms (6-10). Altogether, the use of PLX5622 offers the opportunity to assess the contribution of microglial activity to the neuroinflammatory reaction and potentially represents a new therapeutic approach in stroke. First preclinical pre-conditioning studies have shown PLX5622 treatment to worsen disease outcomes within the first days $(4,11,12)$, increasing infarct size and promoting primary inflammation. However, none of these studies assessed its potential therapeutic effect 
as a long-term treatment. Altogether, this raises the interest of performing a longitudinal study using various imaging, functional and ex vivo assessments to characterise the CSF-1R inhibitor PLX5622-induced changes on stroke-relevant parameters including inflammation, cerebrovascular parameters, and functional outcomes.

To do so, we performed a longitudinal positron emission tomography (PET)/ magnetic resonance (MR) imaging study to non-invasively monitor therapy response, including 18F-DPA714-PET imaging (TSPO, neuroinflammation), $T_{2} w$-weighted $\left(T_{2} w\right.$, lesion), diffusion-weighted (DW, water diffusion) and perfusion-weighted (PW, cerebral blood flow (CBF)) MR imaging. Besides, we performed behavioural tests, including open field (locomotion), pole test (global motor functions), grip strength test (limbs strength) and rotarod (coordination) to assess sensorimotor functions.

We hypothesised that CSF-1R inhibition might represent a new therapeutic intervention in modulating of the microglia-induced post-ischemic inflammation. Long-term CSF-1R inhibition may reduce microglial activity, and therefore decrease the expression of inflammatory markers and improve recovery. The potential impact of CSF-1R inhibition may be non-invasively assessed by combining an extensive multi-parameter PET/MR imaging paradigm with behavioural tests and ex vivo analysis. 


\section{MATERIALS AND METHODS}

\section{Study Approval}

All experiments were conducted in accordance with the German Law on the Care and Use of Laboratory Animals and approved by the Landesamt für Natur, Umwelt und Verbraucherschutz of North Rhine-Westphalia and according to the ARRIVE guidelines (https://www.nc3rs.org.uk/arrive-guidelines).

\section{Study}

In total, $\mathrm{N}=48$ 3-4 months old male C57BL6/6J mice were housed under a standard 12:12 hours light:dark cycle with free access to food and water.

$\mathrm{N}=40$ mice underwent a 30 minutes transient middle cerebral artery occlusion (tMCAo) at day 0 (Figure 1) and were randomised into either control or PLX5622-treated group right after surgery by an external person. Experimenters were blind to group assignment.

All mice underwent $\mathrm{T}_{2} \mathrm{~W}-\mathrm{MRI}$ at day 1 to select animals based on the infarct size. Exclusion criteria were: (i) lack of reperfusion ( $<50 \%$ baseline CBF recovery) assessed by laser Doppler, (ii) infarct exceeding striatal and cortical regions, and (iii) extreme weight loss (> $20 \%$ of the initial body weight). The drop-out rate was $4 \%$.

$\mathrm{N}=8$ mice were used to test the therapy in wild type mice (non stroke) at day 35 post ischemia. Those mice received either control $(n=4)$ or PLX5622 $(n=8)$ diet for 35 days and sacrificed for gene expression analysis. Details on animal groups and numbers are reported in the Supplementary Table 1. 


\section{Experimental Design}

A total of $n=8$ mice/group were used for in vivo PET imaging and behavioural tests. 18FDPA-714-PET imaging were conducted at days 7, 14, 21 and 30 post surgery. Another two groups with $n=8$ mice/group underwent MRI sessions at days 1, 3, 7, 14, 21 and 30 post surgery and included $\mathrm{T}_{2} \mathrm{~W}-, \mathrm{DW}$ - and PW-MRI. PET/MR imaging sessions were performed 12 hours apart. All animals were continuously anaesthetized during surgery and image acquisition. Mice underwent behavioural tests prior and around days 7, 14 and 30 after surgery to assess motor function recovery using open field, rotarod, pole test and the grip test.

At day 35, all animals were anaesthetised and sacrificed by transcardiac perfusion. Brains were harvested for further analysis to assess long-term effects of PLX5622 therapy on the neuroinflammatory response by immunohistochemistry/immunofluorescence. Besides, four groups of $n=4$ mice/group without imaging were added for real-time (RT) qPCR analysis of tissues harvested at day 35 post ischemia.

The experimental timeline is summarised in Figure 1.

\section{Surgery}

Mice underwent a 30 min right tMCAo using an intraluminal occlusion model as previously described with minor modifications (13). Briefly, mice received $0.04 \mathrm{mg}$ Fentanyl/4 mg Midazolam/1g body weight (Fentanyl: RotexMedica, Trittau, Germany; Midazolam: Ratiopharm, Ulm, Germany) prior to surgery. Transient focal cerebral ischemia was induced by introducing a silicone-coated 7-0 monofilament (diameter with coating $0.19 \pm 0.01 \mathrm{~mm}$ ) (Doccol Corporation, 
MA, USA) that was withdrawn after 30 minutes. Mice received a subcutaneous injection of buprenorphine after surgery (0.05-0.1 mg/kg) (Indivior, Berkshire, UK).

\section{Treatment}

PLX5622 was provided by Plexxikon Inc. (USA) and formulated in AIN-76A standard chow by Research Diets Inc. at 1200 ppm chow. Bodyweight was tracked as an index of food intake (Supplementary Figure 1).

\section{F-DPA-714 PET-CT Imaging}

18F-DPA-714 was prepared as previously described with a $>99 \%$ radiochemical purity (14). PET imaging was performed with a high-resolution small animal PET scanner (32 module quadHIDAC, Oxford Positron Systems Ltd., spatial resolution < 1mm FWHM) (15).

The 18F-DPA-714 PET scan was acquired from 45 to 65 minutes post injection of $12.1 \pm 2.0$ MBq (specific activity: 40-80 GBq/ $\mathrm{mmol}$ ). Right after, computed tomography (CT) were acquired using the Inveon CT scanner (Siemens Medical Solutions, spatial resolution: $80 \mu \mathrm{m}$ ).

\section{MR Neuroimaging}

$T_{2}$-weighted MR (repetition time $(T R)=7700 \mathrm{~ms}$, effective echo time $(T E)=100 \mathrm{~ms}$, rare factor $=30$, matrix $=192 \times 192$, averages $=8)$, diffusion-weighted $(T R=2500 \mathrm{~ms}, \mathrm{TE}=31.30 \mathrm{~ms}$; b-values $=100,200,400,600,800,1000,1200,1600,2400\left[\mathrm{~s} / \mathrm{mm}^{2}\right]$, matrix $=128 \times 128$, averages $=8)$ and perfusion-weighted imaging $(T R=10000 \mathrm{~ms}, \mathrm{TE}=5.01 \mathrm{~ms}$, slice $=1$, matrix $=64 \times 64)$ were acquired using a 9.4 T small animal MRI scanner (Biospec 94/20, Bruker Biospin GmbH, Ettlingen, Germany) with a 2 mm-surface coil (Bruker, Ettlingen, Germany) as previously described (16). 


\section{Image Analysis}

Image data were analysed using the in-house developed software MEDgical allowing the analysis of multi-dimensional, multi-scale biomedical image data, as previously described (16).

All the MR images were manually superimposed to the corresponding PET-CT images from the same animal. An atlas-based thresholding approach was used to delineate the infarct volume at day 1 post ischemia (17). Regional 18F-DPA-714 uptake (percentage of injected dose by millilitre, $\% I D / m L)$ was assessed within the $T_{2} w-M R$ based infarct and the contralateral atlasbased striatum.

Similarly, $T_{2} \mathrm{~W}-\mathrm{MR}$ based infarct and its mirrored image were superimposed to the ADC and ASL maps to assess apparent water diffusion coefficient and CBF, respectively. The infarct-tocontralateral ratio was also calculated to account for intra-individual variability.

\section{Immunoreactivity and Quantification}

To assess TSPO cellular source, we performed immunofluorescent TSPO/Iba-1 and TSPO/GFAP co-staining as previously described (16). Additionally, Iba-1/CSF-1R and Iba1/TMEM119 were performed to further characterise the $\mathrm{Iba}-1^{+}$cell population.

For image validation and semi-quantification, we performed TSPO, Iba-1 and GFAP immunostaining as previous described (16). Antibodies are reported in Supplementary Table 2.

Sections were viewed with a combined fluorescent-light microscope (Nikon Eclipse NI-E, Nikon, Tokyo, Japan) and image analysis was performed using Image $1.51 \mathrm{j}$ software (National Institutes of Health, Bethesda, MD, USA). 


\section{Behavioural Tests}

Open field, grip test, rotarod and pole test were performed to evaluate the therapeutic effects of CSF-1R inhibition on motor function recovery during the post ischemic period as previously detailed (16). The four behavioural tests were carried out the week prior to and after surgery, as indicated in Figure 1.

\section{Gene Expression}

Total RNA was isolated from snap-frozen half-brain tissues (RNeasy Mini Kit, Qiagen, Hilden, Germany) and DNAse I treatment (Roche, Mannheim, Germany) to avoid contaminations from genomic DNA. One $\mu \mathrm{g}$ of total RNA was reverse transcribed into first-strand cDNA using the Transcriptor First Strand cDNA Synthesis Kit (Roche, Indianapolis, USA).

The forward and reverse primer sequences (Sigma-Aldrich) are reported in Supplementary Table 3. RT-qPCR was performed using the Rotor-Gene SYBR Green Master mix with the RotorGene $\mathrm{Q}$ device (Qiagen). Relative gene expression was assessed using the $\Delta \Delta \mathrm{Ct}$ method, with Gapdh (Biomol Gmbh, Hamburg, Germany) as a housekeeping gene.

\section{Statistics}

Statistical analysis was performed using SigmaPlot (Systat Software GmbH, Erkrath, Germany). All data were tested for normality and equal variance using the Shapiro-Wilk and Brown-Forsythe tests, respectively. Nonparametric tests were used when assumptions of normality and/or equal variance were not met. In all statistical tests, differences were considered when $p<0.05$. 
Repeated measures (RM) ANOVA, followed by Holm Sidak's post hoc test, was performed for multiple comparisons. Data were expressed as mean \pm standard error of the mean (SEM). For gene expression data analysis, RM ANOVA with Tukey's multiple comparisons was used. For the percentage of stained area, data were displayed as box plot and reported in the respective description as mean \pm standard deviation (SD).

The sample sizes were calculated a priori during the animal ethics dossier application. They were determined based on effect size $(p=0.05$, statistical power: 0.80$)$, mortality rates, and a previous stroke study (18), where we investigated the therapeutic effect of a dietary approach on brain inflammation assessed by 18F-DPA-714 PET imaging study in ischemic mice. We set the minimal detectable difference in means to 0.2 and the expected standard deviation of residuals to 0.1 . 


\section{RESULTS}

For all stroke mice, $\mathrm{T}_{2} \mathrm{~W}-\mathrm{MRI}$ infarct volume significantly decreased over time $(p<0.001)$. No treatment effect was observed ( $p=0.54$ ) (Supplementary Figure 2).

First, we performed longitudinal 18F-DPA-714 PET-CT in both control and PLX5622-treated mice to assess the potential immunomodulatory of PLX5622 (Figure 2A). Individual data are shown in Supplementary Figure 3. Two-way RM ANOVA indicated treatment $(p=0.014$, power: $0.80)$ and treatment*time $(p=0.011)$ effects but no time effect $(p=0.412)$ on tracer uptake within the infarct (Figure 2B). A significant reduction in tracer uptake was observed in PLX5622-treated mice at day 14 post ischemia compared to control mice (control: $2.04 \pm 0.09 \% \mathrm{ID} / \mathrm{mL}, \mathrm{PLX} 5622$ : $1.60 \pm 0.10 \% \mathrm{ID} / \mathrm{mL}, p=0.009)$. Additionally, two-way RM ANOVA indicated a significant effect of treatment ( $p=0.003$, power: 0.89$)$ but not of time $(p=0.227)$ or time*treatment $(p=0.084)$ in the contralateral striatum (Figure 2C). 18F-DPA-714 uptake was significantly decreased in PLX5622 treated mice compared to control mice at days $14(p<0.005), 21(p=0.013)$ and $30(p=$ 0.006) post ischemia, indicative of a permanent drug effect on TSPO levels in non-infarcted tissue. 18F-DPA-714 uptake correlated with TSPO immunoreactivity on brain tissue collected at day 35 post ischemia (Supplementary Figures $4-5, \mathrm{R}^{2}=0.91$ ). No treatment effect was observed on $18 \mathrm{~F}-$ DPA-714 uptake in spleen $(p=0.20)$ and manubrium-sternebra $(p=0.16)$ over time (Supplementary Figure 6).

Furthermore, we tracked therapy response on two stroke-associated MRI parameters, ADC and CBF. Representative DW images and the respective ADC maps from control and PLX5622treated mice are shown in Figure 3A. RM ANOVA indicated main effects of treatment $(p=0.011$, power: 0.55$)$ and time $(p<0.001)$ but not of time*treatment $(p=0.2)$ on ADC within the infarct. 
In control mice, ADC significantly increased at days $21(p=0.022)$ and $30(p=0.006)$ compared to day 1 post ischemia. Similarly, ADC significantly increased at days $21(p=0.10)$ and $30(p=0.003)$ compared to day 3 (Figure 3B). In PLX5622-treated mice, ADC value within the infarct was significantly increased at days $7(p=0.011), 14(p=0.10), 21(p<0.001)$ and $30(p<0.001)$ compared to day 1 . Additionally, ADC were significantly increased at days $21(p=0.016)$ and 30 $(p<0.001)$ compared to day 3. A treatment effect was observed at day 30 post ischemia, where the ADC of PLX5622-treated mice within the infarct was significantly higher than in control mice $(p=0.028)$ (Figure 3B). No treatment effect was observed on ADC values in the contralateral region $(p=0.10)$. The intra-individual follow-up of the infarct-to-contralateral ratio indicated comparable temporal recovery between groups (Supplementary Figure 7).

Representative ASL maps from control and PLX5622-treated mice are shown in Figure 4A. To account for intra-individual variability, we reported the temporal dynamic of the infarct-tocontralateral ratio for both groups (Figure 4B). Two-way RM ANOVA indicated significant effect of time $(p=0.045)$, treatment $(p=0.042$, power: 0.49$)$ and time*treatment $(p=0.019)$. In control mice, the infarct-to-contralateral ratio significantly increased at day 21 compared to day $1(p=$ 0.028) while in PLX5622-treated mice did not show any significant difference over time ( $p=0.15)$. A therapy effect was observed at day 21 , where control mice showed increased infarct-tocontralateral ratio $(1.00 \pm 0.03)$ compared to PLX5622-treated mice $(0.89 \pm 0.03)(p=0.048)$. Mean CBF values are reported in Supplementary Figure 8.

We quantified the amount of Iba- $1^{+}$cells within the infarct, at the periphery of the infarct and in the contralateral striatum of both groups as an index of therapy efficiency (Supplementary Figure 9A). Two-way RM ANOVA indicated treatment $(p<0.001)$ and region $(p<0.001)$ effects. 
Treatment effects were detected at the periphery $(p=0.035)$ and contralateral side $(p<0.001)$, where the percentage of $\mathrm{Iba}-\mathrm{1}^{+}$area was higher in control mice compared to PLX5622-treated mice (Supplementary Figure 9B).

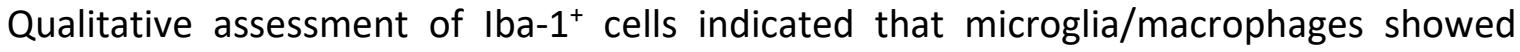
elongated thin processes in control mice, indicative of a resting state, while they showed shorter thicker processes with large soma in PLX5622-treated mice, indicative of a reactive state (Figure 5 and Supplementary Figure 9B). Additionally, the number of ramifications at the periphery and contralateral striatum was higher in control than in PLX5622-treated mice.

Increased astrogliosis was observed in the contralateral striatum of PLX5622-treated mice (Supplementary Figure 10).

We further characterised Iba- $1^{+}$cell population by immunofluorescence. Brain slices from day 35 were co-stained with TSPO, CSF-1R and TMEM119 (Figure 5). Both control and PLX5622 treated mice showed a strong population of Iba- $1^{+} \mathrm{TSPO}^{+}$cells within the infarct, indicating that microglia/macrophages are a TSPO cellular source while most of the GFAP ${ }^{+}$cells (astrocytes) were TSPO- (Supplementary Figure 11). However, we observed many $\mathrm{Iba}-1^{+} \mathrm{CSF}-1 \mathrm{R}^{+}$and Iba$1^{+}$TMEM $119^{+}$cells in control mice while none or few were observed in PLX5622-treated mice. Immunoreactivity was cross-validated by looking at mRNA expression of tspo, csf-1r and tmem119 in wild type and stroke animals (Figure 6A). Tspo, csf-1r, and tmem119 were downregulated after 35-days of treatment $(p<0.005)$ in both wild type and stroke mice, with no significant difference between left and right hemispheres. 
Long-term PLX5622 treatment globally reduced pro-inflammatory $C \times 3 c r 1$ and $C \mathrm{Cl} 2$, and anti-inflammatory Cd4 expression (Figure 6B). Nos2 was only downregulated in the contralateral hemisphere but unaffected in the infarct hemisphere. On the other hand, PLX5622 treatment did not change mRNA expression of pro-inflammatory $\|$ - 16 and tnf and anti-inflammatory Arg1 and II-6 ( $p>0.05)$. Additionally, PLX5622 significantly downregulated the phagocytosis related trem2 gene marker in both hemispheres.

Motor deficits recovery was used as an index of treatment efficiency (Supplementary Figures 12-15). Overall, three of the four tests indicated a treatment effect at day 30 post ischemia. While no significant effect was observed on travelled distance and velocity (open field, $p=0.75)$, PLX5622-treated mice walked shorter distance on the rotarod ( $p=0.023)$, showed less forelimbs strength in the grip test $(p=0.028)$ and moved slower during the pole test $(p=0.041)$ compared to control mice. 


\section{DISCUSSION}

CSF-1R inhibition-induced microglia depletion represents a valuable tool to investigate the contribution of microglia activity to post-ischemic brain injury. For the first time, our study showed the potential of 18F-DPA-714 PET imaging to track the immunomodulatory effect of PLX5622. We found that CSF-1R inhibition transiently decreased radiotracer uptake within the infarct while a sustained decrease was observed in the contralateral healthy tissue. Ex vivo characterisation suggested that one of the major cellular sources of TSPO expression was a potentially therapy-resistant Iba- $1^{+}$cell population. Further characterisation indicated that those cells were mostly CSF-1 $\mathrm{R}^{-}$and TMEM119-, in line with the significant decrease of csf-1r and tmem119 gene expression. We concluded that PLX5622 efficiently inhibited CSF-1R and affected at least the main population of $\mathrm{Iba}-1^{+} \mathrm{CSF}-1 \mathrm{R}^{+}$cells, including $\left(\mathrm{TMEM} 119^{+}\right)$resident microglia, and dampened the expression of some inflammatory markers. Moreover, the contribution from peripheral immune subpopulations to the immune cell pool may be reduced, as indicated by the decrease in $c x 3 c r 1, c c / 2$ and $c d 4$ gene expression. Long-term CSF-1R inhibition also affected homeostatic balance and tissue reperfusion, albeit transient, as indicated by our MRI data.

Altogether, our study highlighted PLX5622 immunomodulatory effect in stroke, which can be non-invasively assessed by 18F-DPA-714 PET imaging. PLX5622 affected subpopulations of microglial cells but did not reduce the total number of Iba- $1^{+}$cells. MRI data supported vascular and homeostasis impairment after long-term treatment.

TSPO-PET is currently the most studied method for spatial measurement and visualization of neuroinflammation, with a bench-to-bed translational value, while other non-TSPO tracers are currently been developed (19). TSPO is markedly upregulated by immune cells such as inner brain 
glial cells (microglia, astrocytes) and peripheral immune cells during inflammatory conditions, making TSPO a suitable biomarker for tracking neuroinflammation and immune cell activation. Among the TSPO tracers, 18F-DPA-714 was used in target validation studies in preclinical and clinical stroke and to track therapy response of immunomodulatory treatment (20) due to its improved bioavailability, specificity and signal-to-noise ratio compared to others TSPO tracers. In humans, image quantification requires patient stratification due to the rs6971 polymorphism causing inter- and intra-individual variability in binding affinity. Established temporal dynamics of 18F-DPA-714 in stroke indicates the number of $\mathrm{CD} 11 \mathrm{~b}^{+} \mathrm{TSPO}^{+} / \mathrm{Iba}^{-} 1^{+} \mathrm{TSPO}^{+}$cells peak around days 11-14, correlating with the peak of radiotracer uptake $(21,22)$. Here, we showed a transient TSPO expression decrease within the infarct in PLX5622-treated mice while TSPO expression was continuously decreased in healthy tissue from day 14 post ischemia. Therefore, we hypothesised that the decrease in 18F-DPA-714 signal around day 14 was potentially caused by the depletion of microglia/macrophages.

Ex vivo characterization revealed a mixed population of $\mathrm{Iba}^{+} 1^{+} / \mathrm{CSF}-1 \mathrm{R}^{-}$and $\mathrm{Iba}-$ $1^{+} /$TMEM119- cells within the infarct with no significant therapy effect on the total number of Iba- $1^{+}$cells. This result indicated that PLX5622 may affect subpopulations of $\mathrm{Iba}-1^{+}$cells, including resident homeostatic TMEM $119^{+}$microglial cells (23), in line with the significantly decreased csf1r, tmem119 and CX3cr1 (myeloid lineage) gene expression. As previously reported (24), PLX5622treated mice also displayed reactive/dystrophic $\mathrm{lba}-1^{+}$cell morphology in perilesional areas compared to control, indicative of a non-resting state. Altogether, changes in protein/gene expression and morphology might indicate a shift in cell functionality, as supported by our gene expression data. 
Additionally, decrease in cx3cr1 (myeloid lineage), ccl2 (monocytes) and cd4 (T cells) gene expression indicated that the peripheral response may be dampened. Similarly, Lei et al. (2020) observed a significant reduction in monocytes, dendritic and T cells of the myeloid/lymphoid compartment in bone-marrow, spleen and blood (25) after long-term treatment. Therefore, further research must considered the role of invading immune cell to the disease phenotype, focusing on (Iba- $\left.1^{+}\right)$perivascular, dendritic and monocytic cells that were not fully depleted with treatment.

Our MRI data indicated that a long-term PLX5622 treatment promotes homeostatic imbalance and impairs vascular integrity, which might partly explain the late functional decline. We propose that the absence of CSF-1 $\mathrm{R}^{+}$cells, including parenchymal and perivascular microglial cells, might transiently impair reperfusion, due to increased vascular leakage (26) and/or atrophy (27).

Therapeutic effects of dietary interventions depends on food intake. The mice showed a significant bodyweight loss within the first days, indicating that therapeutic drug concentration may have been reached only after a few days and potentially acted as a confounding factor.

\section{CONCLUSION}

We demonstrate that long-term CSF-1R inhibition during the post ischemic phase represents an attractive pharmacological tool allowing the timely modulation of the inflammatory microenvironment. Its potential as an immunomodulatory treatment is supported by our longitudinal DPA-714-PET/MRI data. We demonstrate its therapy effect on global TSPO-related inflammation but also detrimental side effects on reperfusion and homeostasis. Further 
investigations are needed to determine the appropriate time window of treatment application to get the maximal therapeutic effect and avoid potential negative impact. Non-invasive imaging techniques allowing intra-individual and longitudinal assessments supporting the identification of specific treatment time intervals.

\section{DISCLOSURE}

The authors declare no conflict of interest.

\section{ACKNOWLEDGEMENTS}

This work was partly funded by the Horizon 2020 Programme under grant agreement $n^{\circ} 675417$ (PET3D), the 'Cells-in-Motion' Cluster of Excellence (DFG EXC1003-CiM), the Herbert-WorchStiftung and the Interdisciplinary Center for Clinical Research (IZKF core unit PIX), Münster.

The authors thank Dirk Reinhardt, Sarah Köster, Christine Bätza, Roman Priebe, Stefanie Bouma, Imgard Hoppe, Christa Möllmann, Nina Knubel and Florian Breuer for their excellent technical support and Franziska Albers and Henriette Lambers for their help with data analysis. 


\section{KEY POINTS}

A. PET/MR imaging as a valuable tool to assess therapeutic microglia modulation after cerebral ischemia.

B. Long-term PLX5622 treatment has immunomodulatory and detrimental effects on stroke as assessed by multi-parametric PET/MRI.

C. PET/MR imaging may allow tracking target-engagement and efficiency of microgliatargeting therapies and refine treatment algorithm. 


\section{REFERENCES}

1. Qin C, Zhou LQ, Ma XT, et al. Dual functions of microglia in ischemic stroke. Neurosci Bull. 2019;35:921-933.

2. Jiang CT, Wu WF, Deng YH, Ge JW. Modulators of microglia activation and polarization in ischemic stroke (Review). Mol Med Rep. 2020;21:2006-2018.

3. Chitu V, Gokhan Ş, Nandi S, Mehler MF, Stanley ER. Emerging roles for CSF-1 receptor and its ligands in the nervous system. Trends Neurosci. 2016;39:378-393.

4. Elmore MRP, Najafi AR, Koike MA, et al. Colony-stimulating factor 1 receptor signaling is necessary for microglia viability, unmasking a microglia progenitor cell in the adult brain. Neuron. 2014;82:380-397.

5. Rice RA, Pham J, Lee RJ, Najafi AR, West BL, Green KN. Microglial repopulation resolves inflammation and promotes brain recovery after injury. Glia. 2017;65:931-944.

6. Nissen JC, Thompson KK, West BL, Tsirka SE. Csf1R inhibition attenuates experimental autoimmune encephalomyelitis and promotes recovery. Exp Neurol. 2018;307:24-36.

7. Beckmann N, Giorgetti E, Neuhaus A, et al. Brain region-specific enhancement of remyelination and prevention of demyelination by the CSF1R kinase inhibitor BLZ945. Acta Neuropathol Commun. 2018;6:9.

8. Dagher NN, Najafi AR, Kayala KMN, et al. Colony-stimulating factor 1 receptor inhibition prevents microglial plaque association and improves cognition in 3xTg-AD mice. $J$ Neuroinflammation. 2015;12:1-14. 
9. Spangenberg EE, Lee RJ, Najafi AR, et al. Eliminating microglia in Alzheimer's mice prevents neuronal loss without modulating amyloid- $\beta$ pathology. Brain. 2016;139:12651281.

10. Unger MS, Schernthaner P, Marschallinger J, Mrowetz H, Aigner L. Microglia prevent peripheral immune cell invasion and promote an anti-inflammatory environment in the brain of APP-PS1 transgenic mice. J Neuroinflammation. 2018;15:1-23.

11. Szalay G, Martinecz B, Lénárt N, et al. Microglia protect against brain injury and their selective elimination dysregulates neuronal network activity after stroke. Nat Commun. 2016;7:11499.

12. Otxoa-de-Amezaga A, Miró-Mur F, Pedragosa J, et al. Microglial cell loss after ischemic stroke favors brain neutrophil accumulation. Acta Neuropathol. 2019;137:321-341.

13. Engel O, Kolodziej S, Dirnagl U, Prinz V. Modeling stroke in mice - Middle cerebral artery occlusion with the filament model. J Vis Exp. 2011;47:2423.

14. James ML, Fulton RR, Vercoullie J, et al. DPA-714, a new translocator protein-specific ligand: Synthesis, radiofluorination, and pharmacologic characterization. J Nucl Med. 2008;49:814-822.

15. Schäfers KP, Reader AJ, Kriens M, Knoess C, Schober O, Schäfers M. Performance evaluation of the 32-module quadHIDAC small-animal PET scanner. J Nucl Med. 2005;46:996-1004.

16. Barca C, Wiesmann M, Calahorra J, et al. Impact of hydroxytyrosol on stroke: tracking therapy response on neuroinflammation and cerebrovascular parameters using PET-MR 
imaging and on functional outcomes. Theranostics. 2021;11:4030-4049.

17. Barca C, Foray C, Hermann S, et al. Characterization of the inflammatory post-ischemic tissue by full volumetric analysis of a multimodal imaging dataset. Neuroimage. 2020;222:117217.

18. Wiesmann $M$, Zinnhardt $B$, Reinhardt $D$, et al. A specific dietary intervention to restore brain structure and function after ischemic stroke. Theranostics. 2017;7:493-512.

19. Jain P, Chaney AM, Carlson ML, Jackson IM, Rao A, James ML. Neuroinflammation PET imaging: current opinion and future directions. J Nucl Med. 2020;61:1107-1112.

20. Zinnhardt $B$, Wiesmann $\mathrm{M}$, Honold $\mathrm{L}$, et al. In vivo imaging biomarkers of neuroinflammation in the development and assessment of stroke therapies - towards clinical translation. Theranostics. 2018;8:2603-2620.

21. Martín A, Boisgard R, Thézé B, et al. Evaluation of the PBR/TSPO radioligand 18F-DPA-714 in a rat model of focal cerebral ischemia. J Cereb Blood Flow Metab. 2010;30:230-241.

22. Zinnhardt B, Viel T, Wachsmuth $\mathrm{L}$, et al. Multimodal imaging reveals temporal and spatial microglia and matrix metalloproteinase activity after experimental stroke. J Cereb Blood Flow Metab. 2015;35:1711-1721.

23. Bennett ML, Bennett FC, Liddelow SA, et al. New tools for studying microglia in the mouse and human CNS. Proc Natl Acad Sci. 2016;113:E1738-E1746.

24. Kerkhofs D, Van Hagen BT, Milanova I V., et al. Pharmacological depletion of microglia and perivascular macrophages prevents Vascular Cognitive Impairment in Ang II-induced 
hypertension. Theranostics. 2020;10:9512-9527.

25. Lei F, Cui N, Zhou C, Chodosh J, Vavvas DG, Paschalis EI. CSF1R inhibition by a smallmolecule inhibitor is not microglia specific; Affecting hematopoiesis and the function of macrophages. Proc Natl Acad Sci U S A. 2020;117:23336-23338.

26. Halder SK, Milner R. A critical role for microglia in maintaining vascular integrity in the hypoxic spinal cord. Proc Natl Acad Sci U S A. 2019;116:26029-26037.

27. Yang $X$, Zhao L, Campos MM, et al. CSF1R blockade induces macrophage ablation and results in mouse choroidal vascular atrophy and RPE disorganization. Elife. 2020;9:e55564. 


\section{Behavioural tests}

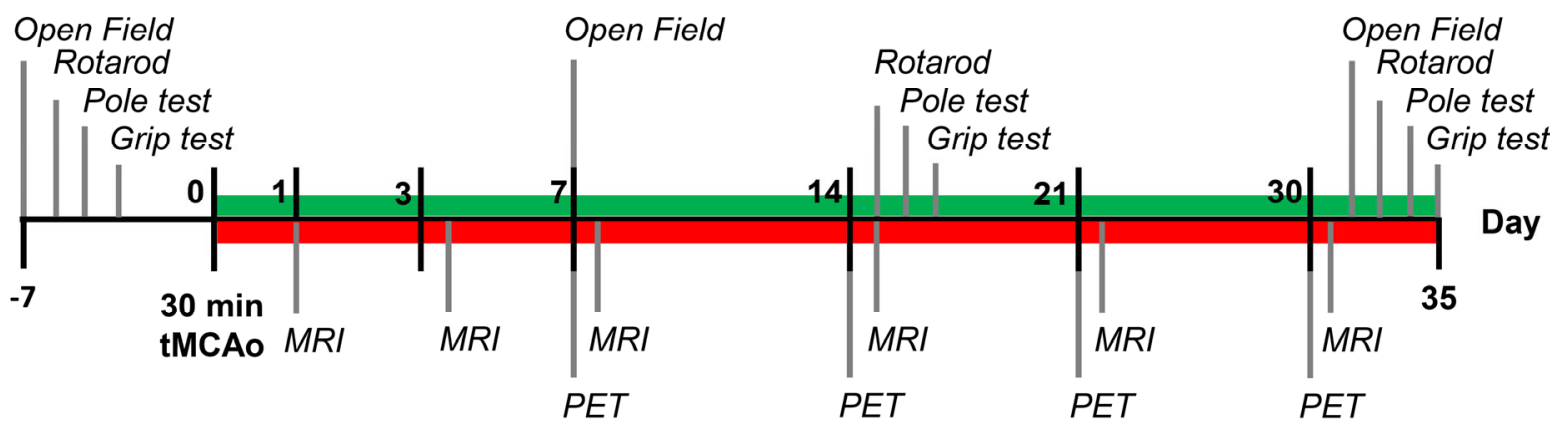

In vivo imaging
Control
PLX5622

Figure 1. Study design. 

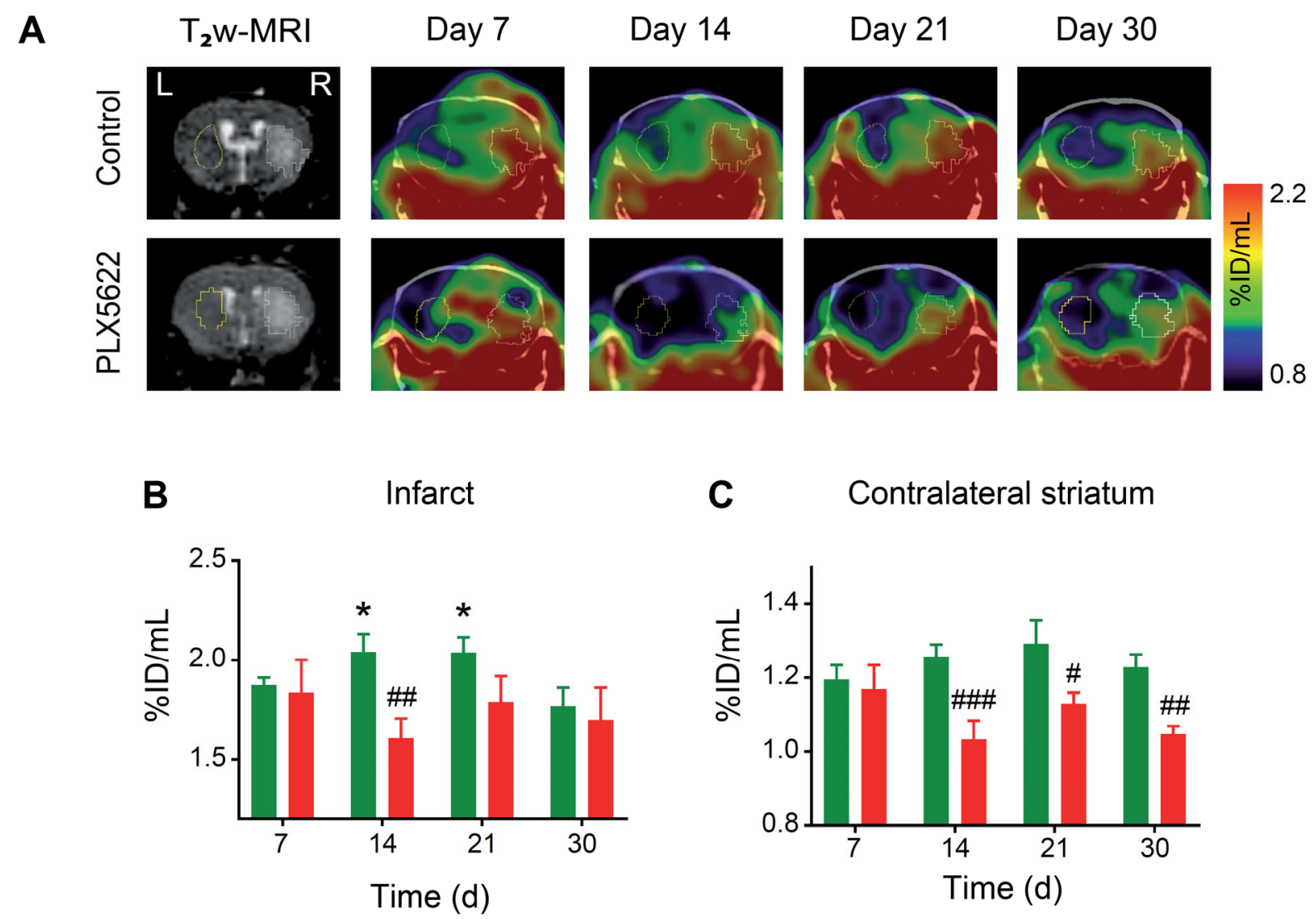

Control

PLX5622

Figure 2. 18F-DPA-714 PET-CT imaging. (A) Representative 18F-DPA-714 (TSPO) PET-CT images and corresponding day-1 $\mathrm{T}_{2} \mathrm{~W}-\mathrm{MR}$ image. (B) Quantification of $18 \mathrm{~F}-\mathrm{DPA}-714$ uptake (\%ID/mL) within the infarct and (C) the contralateral striatum $\left({ }^{*} p<0.05,{ }^{* *} p<0.01,{ }^{* *} p<0.005\right.$; ${ }^{*}$ vs day 30 post ischemia, \# vs treatment). 
A
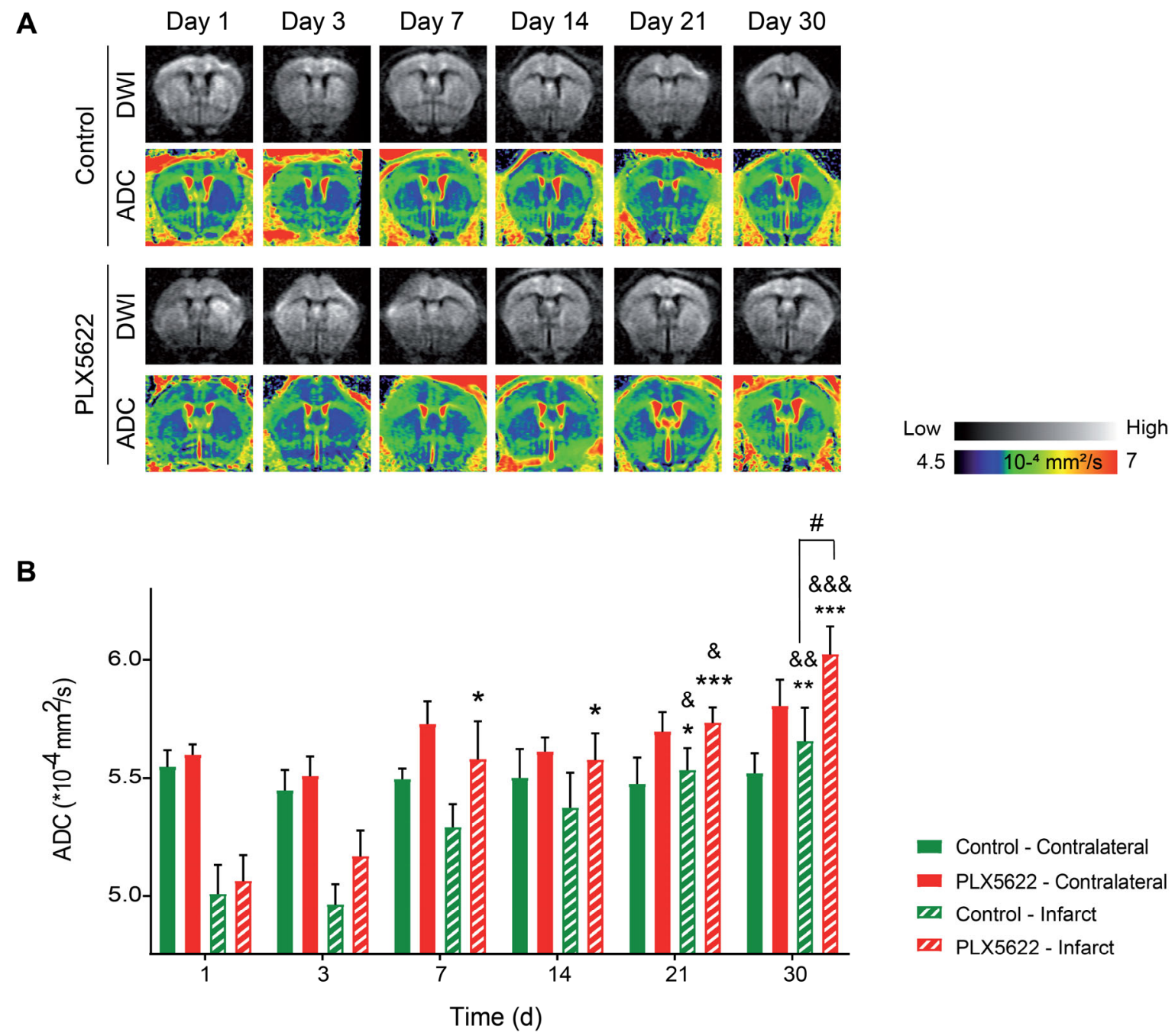

- Control - Contralateral

- PLX5622 - Contralateral

E Control - Infarct

E PLX5622 - Infarct

Figure 3. $A D C$ map. (A) Representative $D W$ images (for $b=2400 \mathrm{~s} / \mathrm{mm}^{2}$ ) and the respective $A D C$ maps. (B) The time course of the ADC value indicated homeostatic imbalance within the infarct at day 30 with treatment $\left({ }^{*} p<0.05,{ }^{* *} p<0.01,{ }^{* * *} p<0.005, *\right.$ vs day $1, \&$ vs day $3, \$$ vs day $7, \#$ vs treatment). 
A $\quad \mathrm{T}_{2 \mathrm{~W}} \mathrm{MRI}$

Day 1
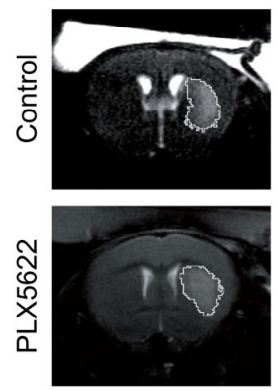

B

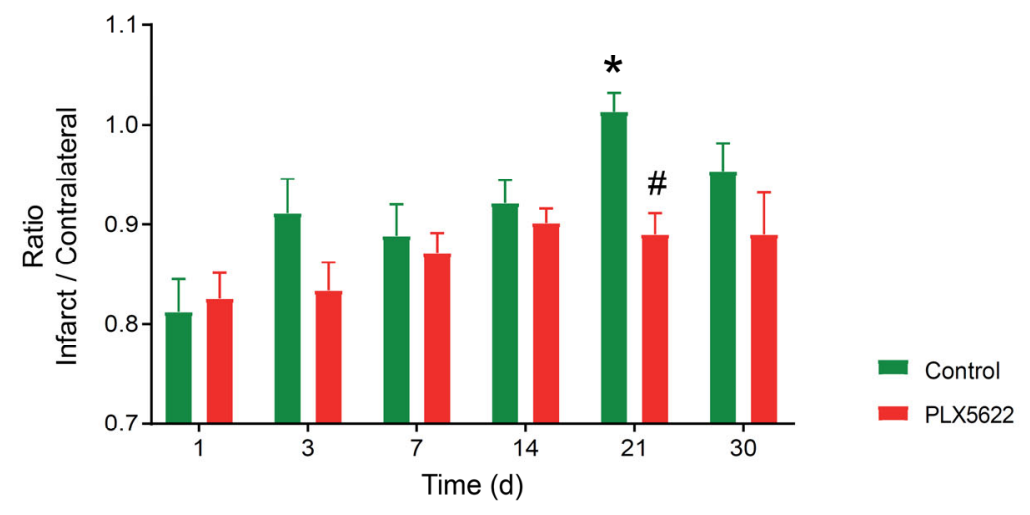

Figure 4. ASL map. (A) Representative ASL maps and the corresponding day-1 $T_{2} W-M R$ image. (B) PLX5622-treated mice showed a significant lower infarct-to-contralateral ratio than control mice at day 21 , indicative of impaired tissue reperfusion within the infarct $\left({ }^{*} p<0.05, *\right.$ vs day $1, \#$ vs treatment). 

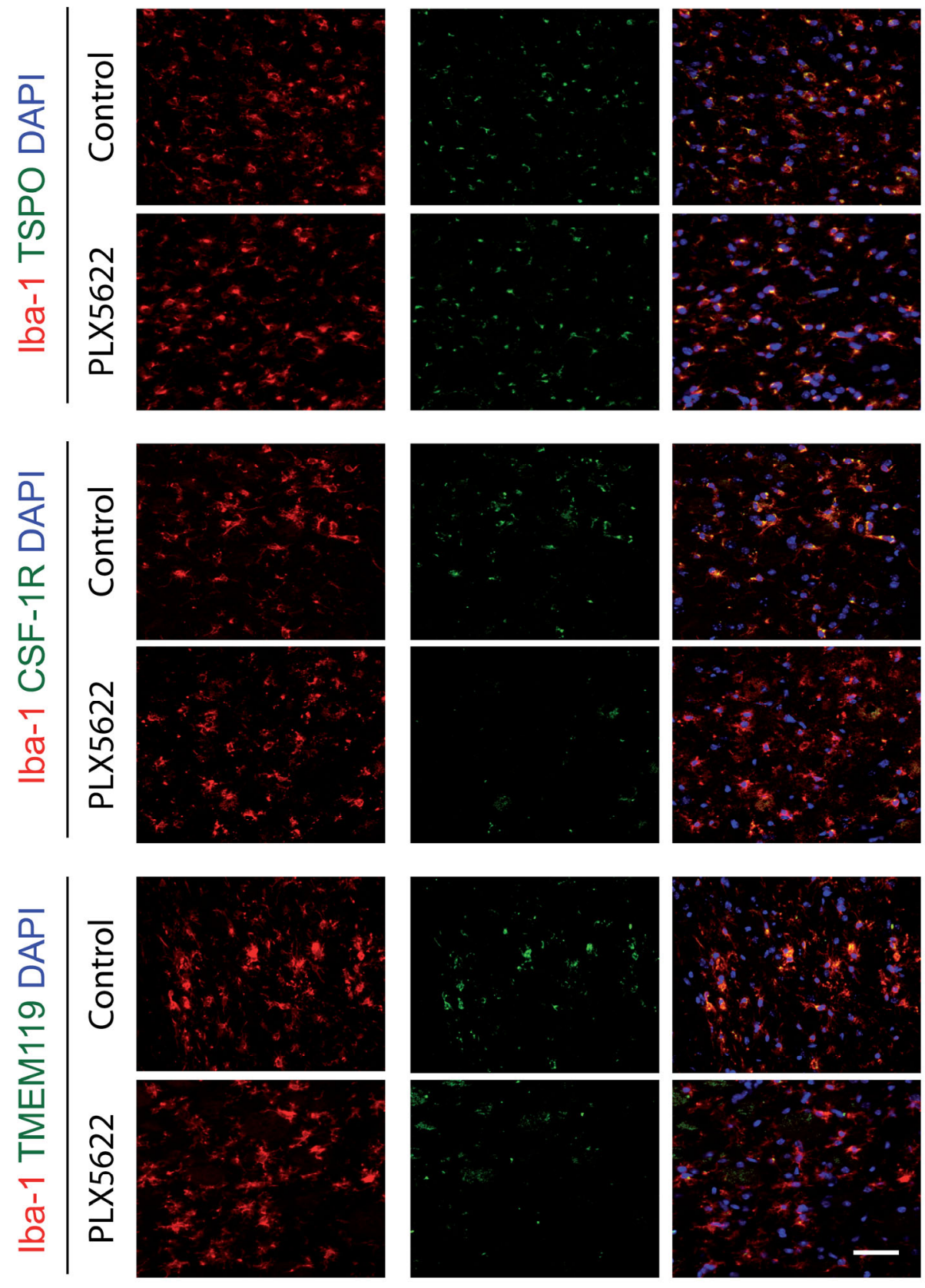

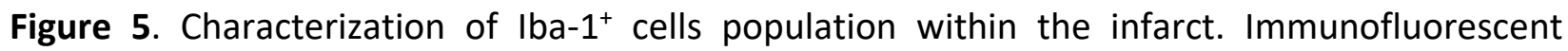
staining for TSPO, CSF-1R and TMEM119 in Iba-1+ cell population in both control and PLX5622treated mice at day 35 post ischemia. Scale bar: $15 \mu \mathrm{m}$. 


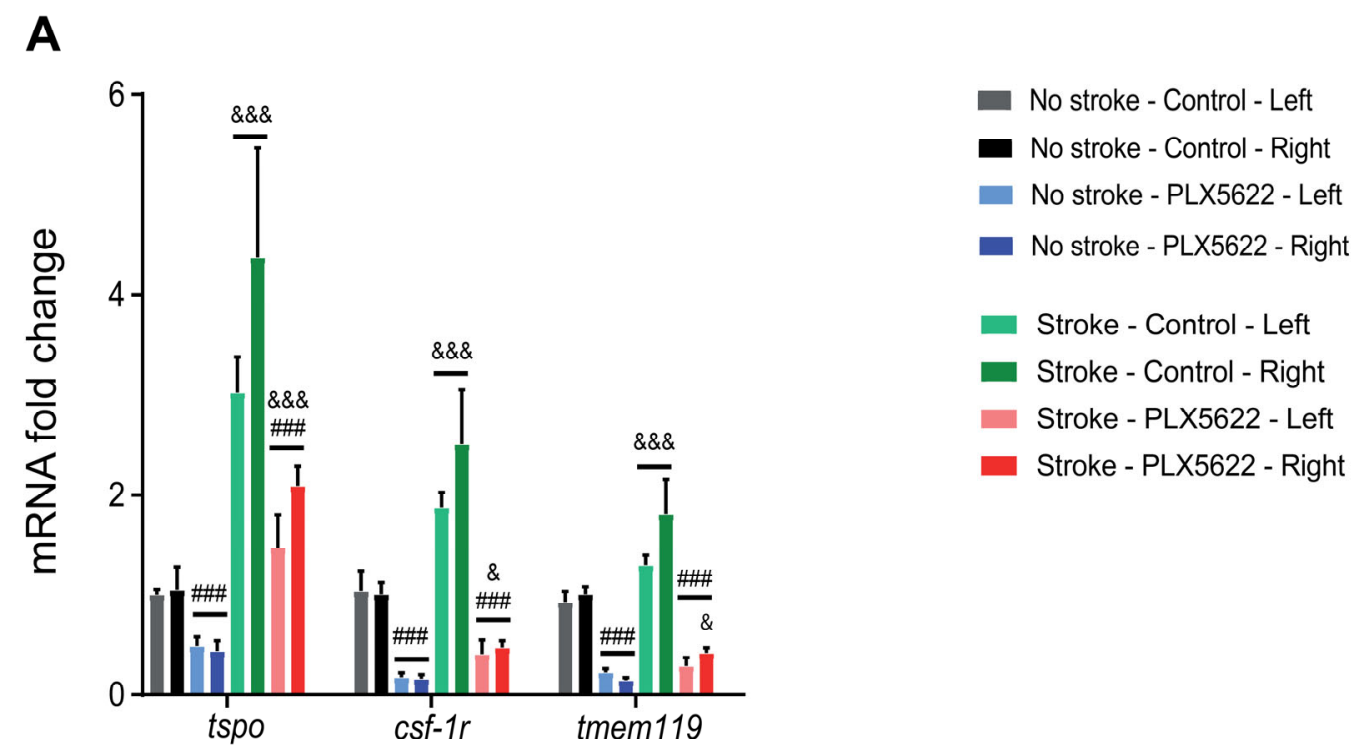

B

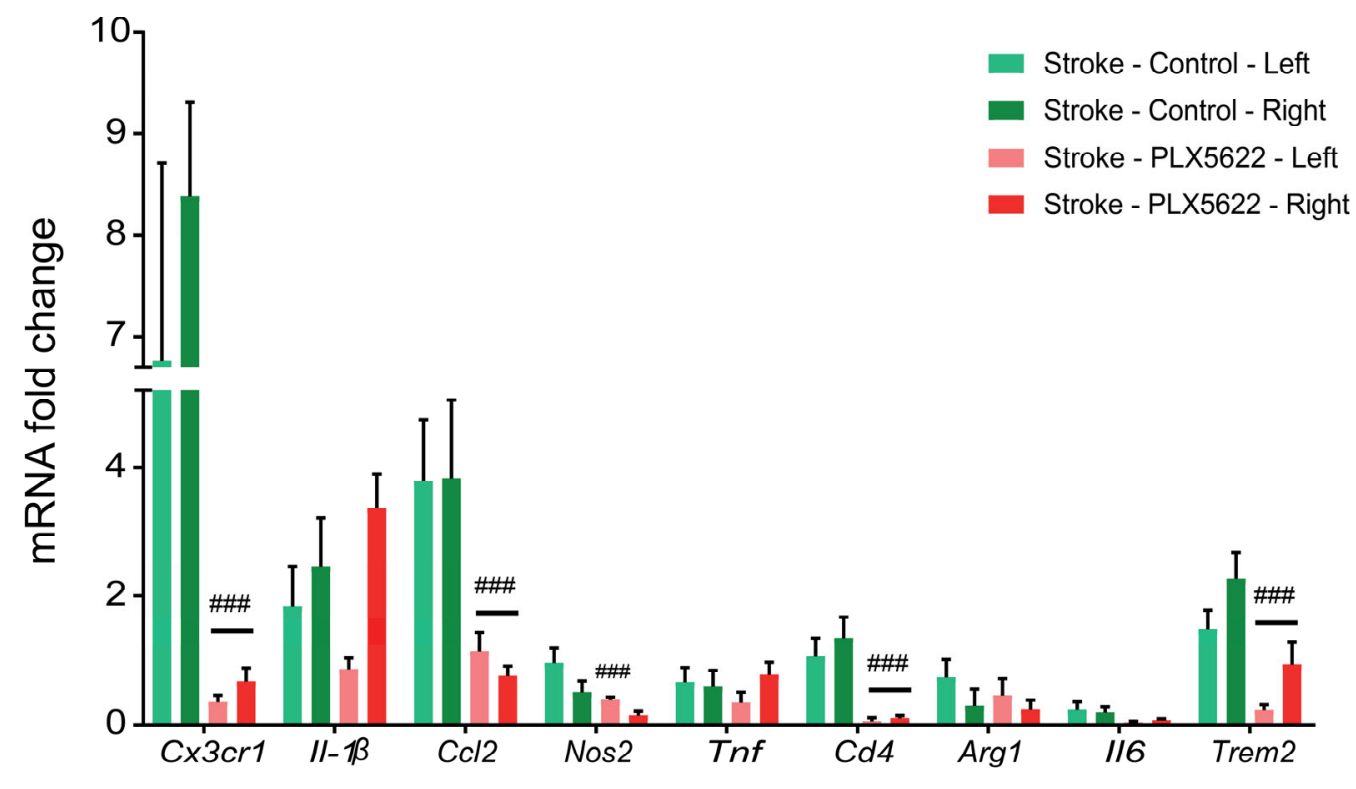

Figure 6. Gene expression. (A) Tspo, csf-1r and tmem119 expression significantly decreased with treatment in both wild type and stroke animals. (B) Gene transcription was efficiently downregulated for several pro-inflammatory, anti-inflammatory and phagocytosis-relevant genes upon CSF-1R inhibition ( ${ }^{*} p<0.05,{ }^{* *} p<0.01,{ }^{* *} p<0.005$; \# vs treatment, \& vs wild type mice). 


\section{Supplementary files}

Supplementary Table 1. Study design and animal number. All in vivo PET imaging and behavioural tests were performed on $n=8$ stroke mice per group (groups $A \& E$ ). Another $n=8$ stroke mice per group were used for MR imaging (groups B \& F). At day 35 post ischemia, those animals were sacrificed and used for histological characterization of the inflammatory.

Besides, four groups of $n=4$ mice per group without imaging were added for gene expression analysis of tissues harvested at day 35 post ischemia (group C: wild type with control diet, group G: wild type with PLX5622 diet, group D: stroke with control diet and group H: stroke with PLX5622 diet).

\begin{tabular}{|c|c|c|c|c|c|}
\hline Diet & Group & Tmcao & $\begin{array}{c}\text { PET \& } \\
\text { Behaviour }\end{array}$ & MRI & $\begin{array}{c}\text { Ex vivo } \\
\text { only }\end{array}$ \\
\hline \multirow{4}{*}{$\begin{array}{l}\text { Control } \\
(n=24)\end{array}$} & $A$ & Yes & 8 & - & - \\
\hline & $B$ & Yes & - & 8 & - \\
\hline & $C$ & No & - & - & 4 \\
\hline & $D$ & Yes & - & - & 4 \\
\hline \multirow{4}{*}{$\begin{array}{l}\text { PLX5622 } \\
(n=24)\end{array}$} & $E$ & Yes & 8 & - & - \\
\hline & $\mathrm{F}$ & Yes & - & 8 & - \\
\hline & $\mathrm{G}$ & No & - & - & 4 \\
\hline & $\mathrm{H}$ & Yes & - & - & 4 \\
\hline
\end{tabular}


Supplementary Table 2. List of antibodies used for immunohistochemistry and immunofluorescence.

Primary antibodies

\begin{tabular}{|l|l|l|l|l|}
\hline Target & Dilution & Species & ID & Provider \\
\hline Anti-PBR & $1: 250$ & Rabbit & ab109497 & Abcam \\
\hline $\begin{array}{l}\text { Recombinant Alexa } \\
\text { Fluor 488 anti-PBR }\end{array}$ & $1: 250$ & Rabbit & ab199779 & Abcam \\
\hline Anti-Iba-1 & $1: 500$ & Rabbit & $019-19741$ & Wako \\
\hline $\begin{array}{l}\text { Red fluorochrome } \\
\text { (635)-conjugated Iba-1 }\end{array}$ & $1: 500$ & Rabbit & $013-26471$ & Wako \\
\hline Anti-CSF-1R & $1: 250$ & Rabbit & SAB4500498 & Sigma-Aldrich \\
\hline Anti-GFAP & $1: 500$ & Chicken & ab4675 & Abcam \\
\hline Anti-TMEM119 & $1: 200$ & Rabbit & ab209064 & Abcam \\
\hline
\end{tabular}

\section{Secondary antibodies}

\begin{tabular}{|l|l|l|l|l|}
\hline Target & Dilution & Species & ID & Provider \\
\hline Biotin Anti-rabbit & $1: 800$ & Goat & B21078 & Life Technologies \\
\hline Biotin Anti-chicken & $1: 800$ & Goat & D20701 & Life Technologies \\
\hline
\end{tabular}

\begin{tabular}{|l|l|l|l|l|}
\hline Alexa Fluor 488 anti-rabbit & $1: 1000$ & Goat & A-21206 & Life Technologies \\
\hline Alexa Fluor 555 anti-rabbit & $1: 1000$ & Goat & A-21432 & Life Technologies \\
\hline Alexa Fluor 488 anti-chicken & $1: 1000$ & Goat & A-11039 & Life Technologies \\
\hline
\end{tabular}

Abbreviations: CSF-1R: colony stimulating factor-1 receptor; GFAP: glial fibrillary acidic protein;

Iba-1: ionized calcium binding adapter molecule-1; PBR: peripheral benzodiazepine receptor;

TMEM119: transmembrane protein 119. 
Supplementary Table 3. Primers for real time qPCR.

\begin{tabular}{|c|c|c|}
\hline Primer & Forward & Reverse \\
\hline Csf-1r & gccatatacaggtacacattc & gtgccattaagaagtactgg \\
\hline$T s p o$ & ggatctttccagaacatcag & acgtacaaagtaggctcc \\
\hline$C d 4$ & tagcaactctaaggtctctaac & gatagctgtgctctgaaaac \\
\hline IIb & ggatgatgatgataacctgc & catggagaatatcacttgttgg \\
\hline II6 & aagaaatgatggatgctacc & gagtttctgtatctctctgaag \\
\hline Arg1 & ctgacctatgtgtcatttgg & catctgggaactttcctttc \\
\hline Tmem119 & ccagagctggttccatag & gggagtgacacagagtag \\
\hline Cx3cr1 & aacaccatgctgtcatattc & gtaagctactatgcttgctg \\
\hline ccl2 & caagatgatcccaatgagtag & ttggttgacaaaaactacagc \\
\hline Nos2 & catcaaccagtattatggctc & tttcctttgttacagcttcc \\
\hline Tnf & ctatgtctcagcctcttctc & catttgggaacttctcatcc \\
\hline Trem2 & tcatctctttctgcacttc & tcataagtacatgacaccctc \\
\hline Gfap & ggaagatctatgaggaggaag & ctgcaaacttagaccgatac \\
\hline Gapdh & ctggagaaacctgccaagta & tgttgctgtagccgtattca \\
\hline
\end{tabular}

Abbreviations: CSF-1R: Colony stimulating factor-1 receptor; Tspo: Translocator protein, Cd4:

Cluster of differentiation 4; Il1b: Interleukin-16; II6: Interleukin-6; Arg1: Arginase 1; Tmem119:

Transmembrane protein 119; Cx3cr1: C-X3-C chemokine receptor 1; Ccl2: Chemokine C-C ligand 2;

Nos2: Nitric oxide synthase 2; Tnf: Tumor necrosis factor; Trem2: Triggering receptor expressed on myeloid cells 2; Gfap: Glial fibrillary acidic protein; Gapdh: Glyceraldehyde 3-phosphate dehydrogenase. 


\section{Supplementary figures}

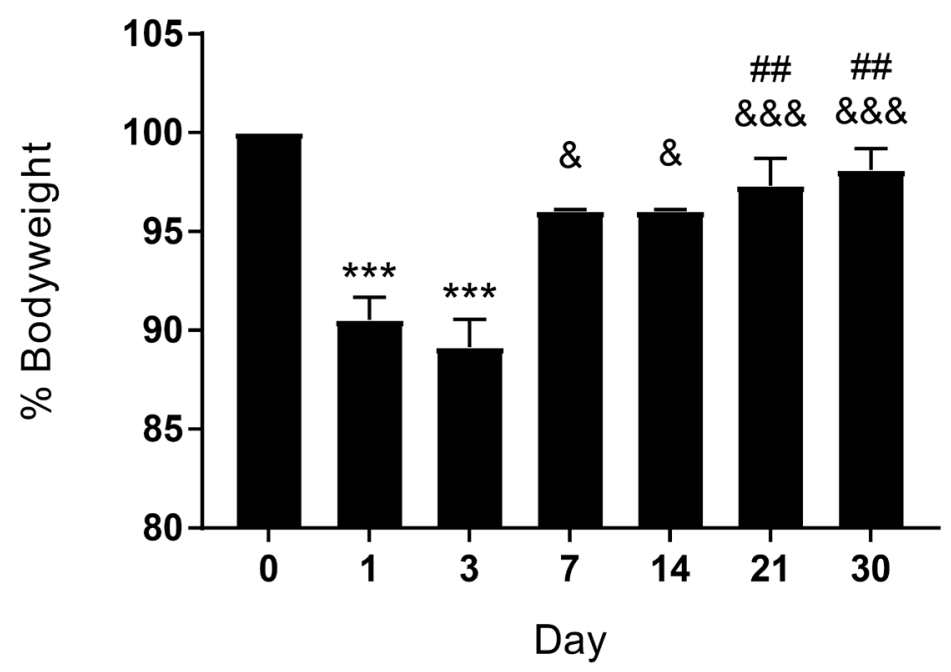

Supplementary Figure 1. Bodyweight. Two-way RM ANOVA indicated a significate effect of time $(p<0.01)$ but not of treatment $(p=0.20)$ on bodyweight. Friedman repeated measures ANOVA on ranks indicated that bodyweight significantly decreased after surgery within the first days after stroke, indicative of reduced food intake.

Statistical analysis was carried out with Friedman RM ANOVA followed by Tukey's post hoc test for multiple comparisons $(n=16)$. Data are expressed as mean $\pm \operatorname{SEM}\left({ }^{*} p<0.05,{ }^{* *} p<0.01,{ }^{* * *} p\right.$ $<0.005 ;{ }^{*}$ vs day 0 (baseline), \# vs day 1 and \& vs day 3 ). 


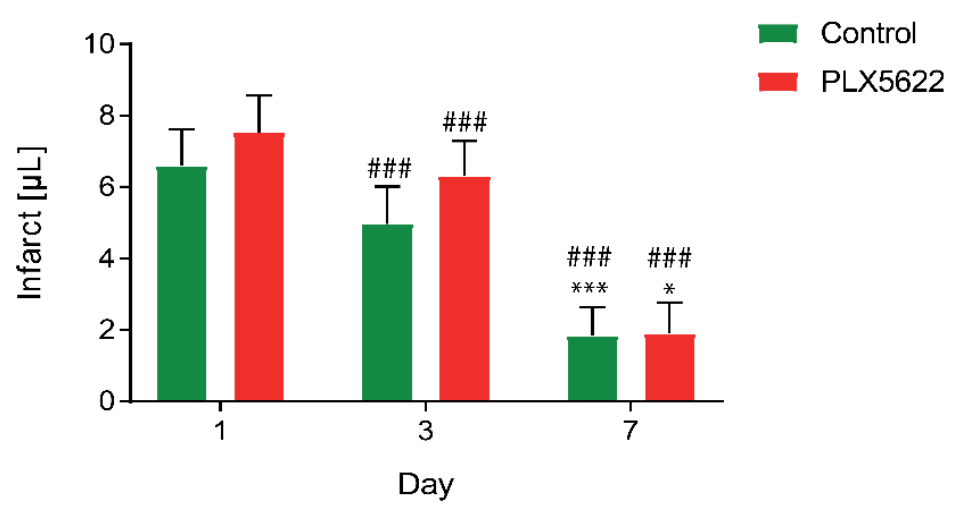

\section{Supplementary Figure 2. PLX5622 treatment does not affect $\mathrm{T}_{2} \mathrm{~W}-\mathrm{MRI}$ infarct volume.}

Quantification of $\mathrm{T}_{2} \mathrm{~W}-\mathrm{MRI}$ infarct volume at days 1, 3 and 7 after tMCAo for both experimental groups (green: control, red: PLX5622) showed decreased infarct volume over time. No treatment effect was observed $(p=0.54)$.

Data were analysed with a two-way RM ANOVA followed by Holm Sidak's post hoc test for multiple comparisons. Data are depicted as mean \pm SEM $(n=8 /$ group) $(* p<0.05, * * p<0.01$, $* * * p<0.005 ; \#$ vs day $1,{ }^{*}$ vs day 3$)$. 


\section{A. $T_{2} W-M R I$ infarct}

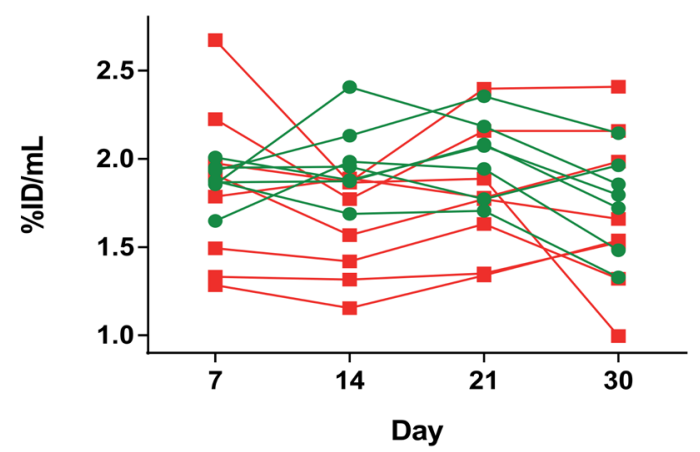

B. Contralateral striatum

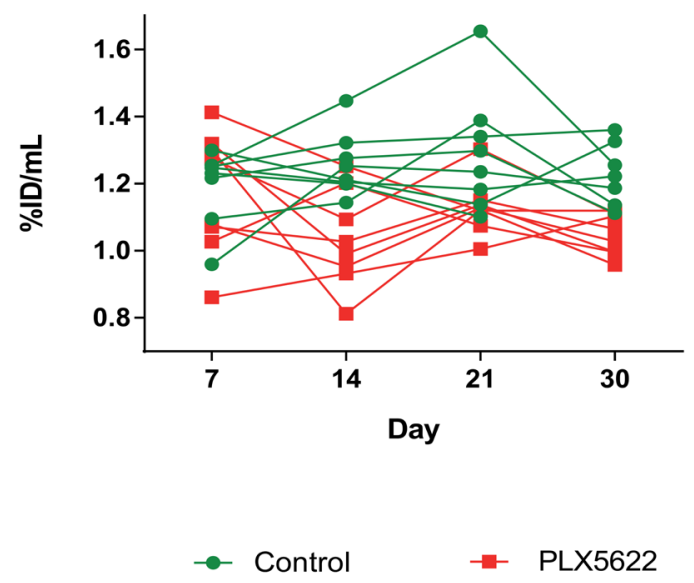

Supplementary Figure 3. Individual 18F-DPA-714 radiotracer uptake over time. Two-way RM ANOVA indicated a treatment $(p=0.014)$ and treatment*time $(p=0.011)$ effect but no time effect $(p=0.412)$ on tracer uptake within the infarct. Data was analysed with a two-way RM ANOVA followed by Holm Sidak's post hoc test for multiple comparisons. Data are depicted as \%ID/mL ( $\mathrm{n}$ = 8/group, green line: control, red line: PLX5622-treated mice). 
A.
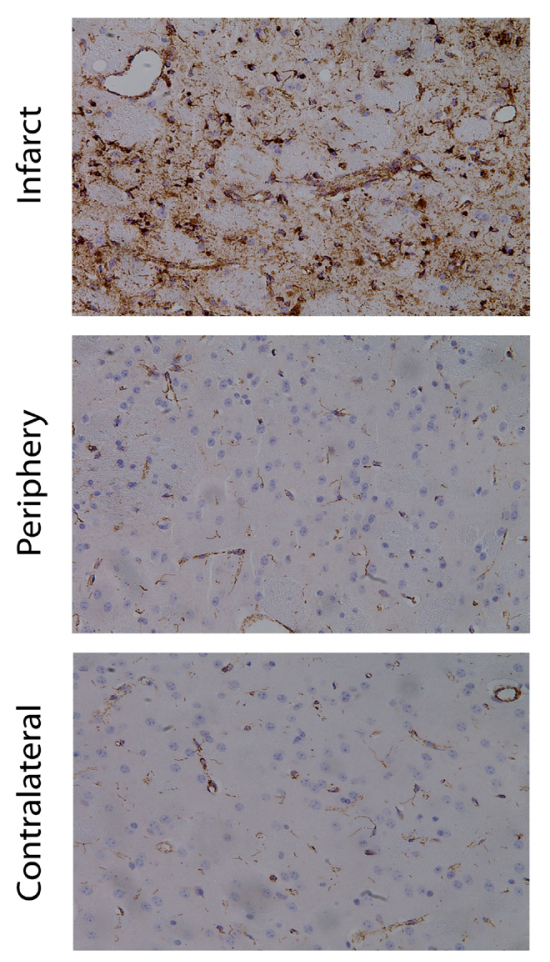

B.

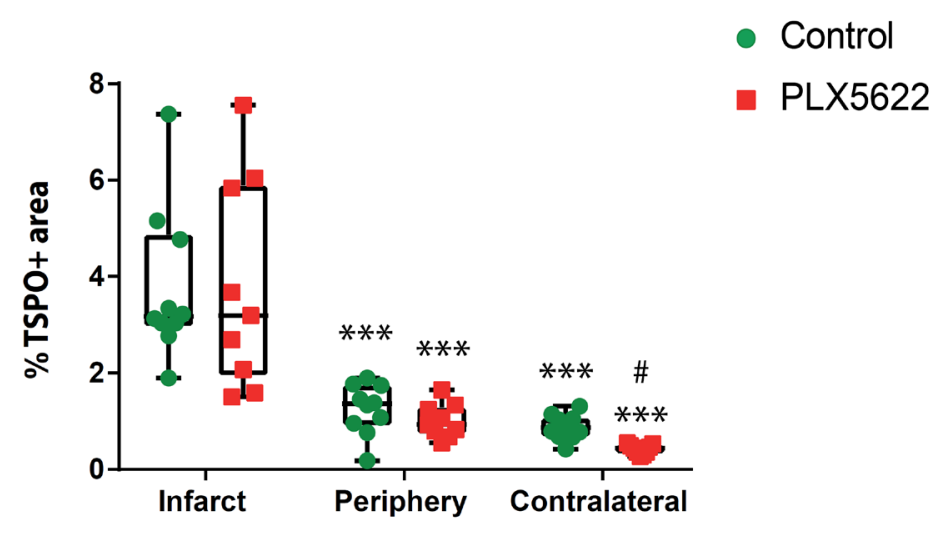

PLX5622
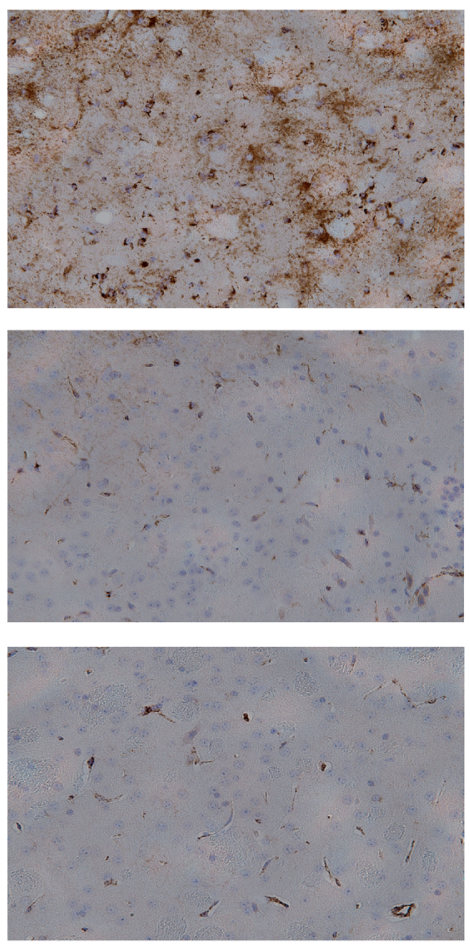

था

\section{Supplementary Figure 4. Quantification of TSPO+ area validated 18F-DPA-714 imaging at day 35}

post ischemia. (A) The representative images of the anti-TSPO immunoreactivity showing TSPO ${ }^{+}$ cells and vessels within the infarct, at the periphery of the infarct and in contralateral striatum at day 35 post ischemia of both control $(n=4)$ and PLX5622-treated mice $(n=3)$. (B) Quantification of the percentage of $\mathrm{TSPO}^{+}$area in the 3 regions of interest showed significant decreased of $\mathrm{TSPO}^{+}$ area in PLX5622 mice compared to control mice only in the contralateral striatum, confirming 
18F-DPA-714 PET images acquired at day 30 post ischemia. Data was analysed by two-way RM ANOVA followed by Sidak's post hoc test $\left({ }^{*} p<0.05,{ }^{* *} p<0.01,{ }^{* * *} p<0.005{ }^{*}\right.$ vs infarct, \# vs diet). 


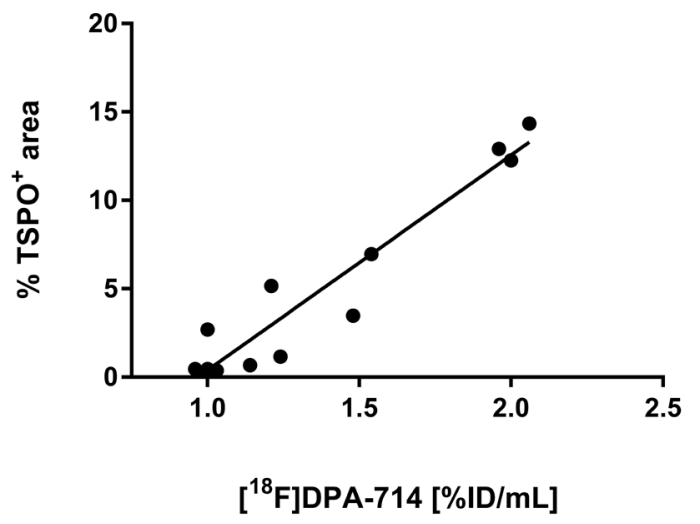

Supplementary Figure 5. Cross-validation of 18F-DPA-714 PET images by TSPO immunoreactivity at day 35 post ischemia $\left(R^{2}=0.91\right)$. 
A

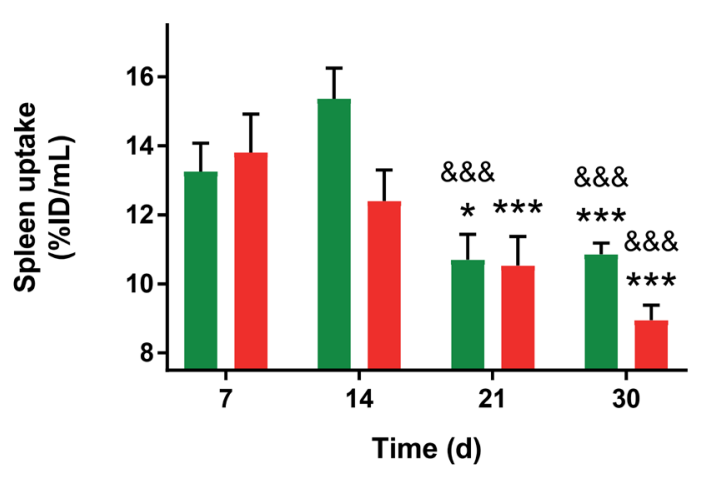

B

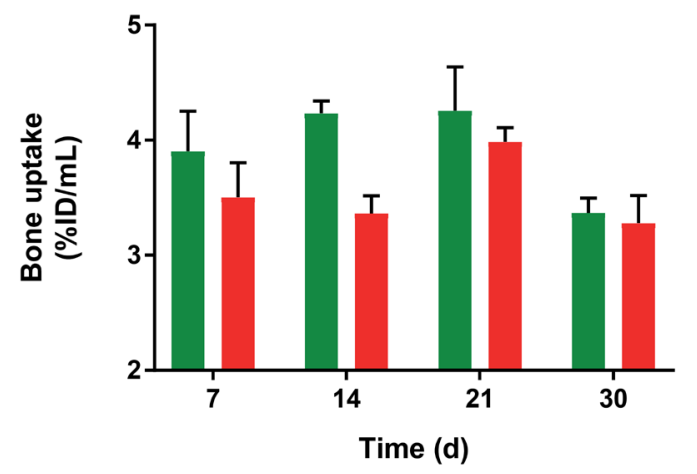

\section{Control PLX5622}

Supplementary Figure 6. Spleen and bone 18F-DPA-714 tracer uptake. (A) The dataset passed the normality $(p=0.25)$ and equal variance $(p=0.32)$ tests. Two-way RM ANOVA indicated a significant effect of time $(p<0.001$, power $=0.98)$ but not of treatment $(p=0.20$, power $=0.13)$ or time*treatment $(p=0.09)$ on spleen tracer uptake $(n=8 /$ group). Spleen uptake was significantly decreased from days $7 / 14$ to days $21 / 30$ post ischemia. (B) Two-way RM ANOVA indicated neither effect of time $(p=0.56)$ or treatment $(p=0.16)$ on bone tracer uptake. An indicative treatment effect could be observed at day 14 on both spleen and bone tracer uptake while not significant.

The volumes-of-interest were manually delineated on the CT scans and co-registered PET images. The bone region included the manubrium and the first connecting sternebra). Data are expressed as mean $\pm \operatorname{SEM}\left({ }^{*} p<0.05,{ }^{* *} p<0.01,{ }^{* * *} p<0.005 ;{ }^{*}\right.$ vs day $7, \&$ vs day $14, \#$ vs treatment). 


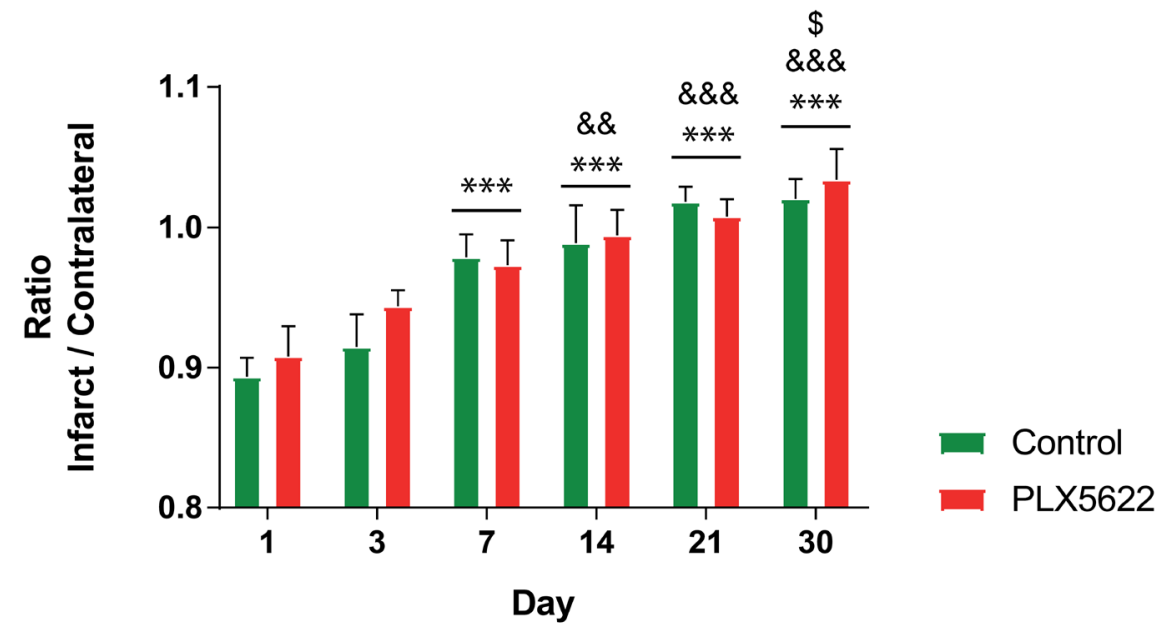

Supplementary Figure 7. Temporal dynamics of the infarct-to-contralateral ADC ratio within the infarct and contralateral striatum in control and PLX5622-treated mice. Two-way RM ANOVA analysis indicated significant effect of time $(p<0.001)$ but not of treatment $(p=0.589)$ or time*treatment $(p=0.899)$ on the infarct-to-contralateral ADC ratio. Data was analysed with a two-way RM ANOVA followed by Holm Sidak's post hoc test for multiple comparisons. Data are depicted as mean \pm SEM ( $n=8 /$ group) $\left({ }^{*} p<0.05,{ }^{* *} p<0.01, * * * p<0.005,{ }^{*}\right.$ vs day $1, \&$ vs day $3, \$$ vs day 7). 


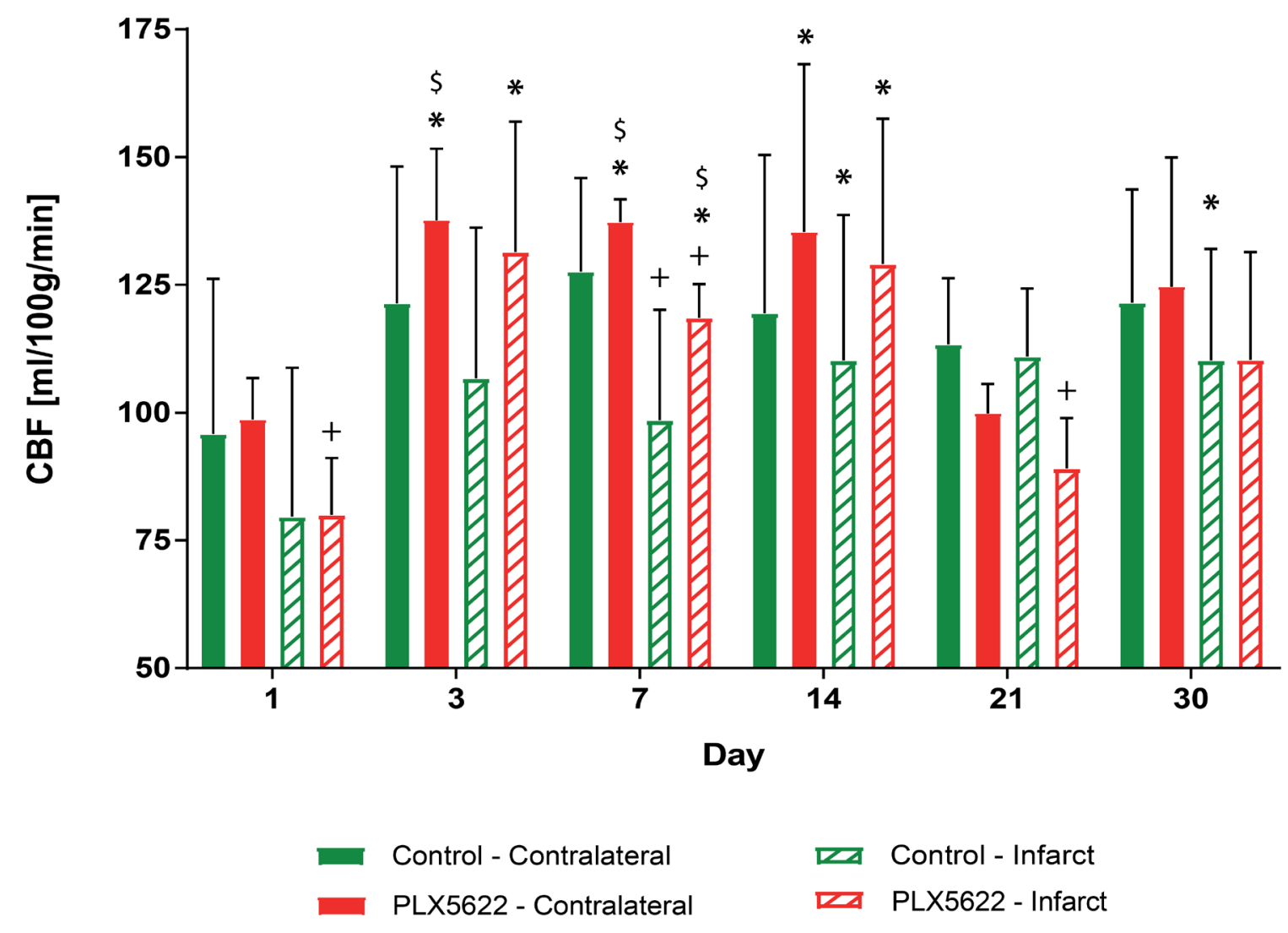

Supplementary Figure 8. Temporal dynamics of CBF mean values within the infarct and contralateral striatum in control and PLX5622 treated mice. In control mice, no significant difference in CBF values was observed in the contralateral side over time $(p>0.05)$.

Mean CBF values within the infarct at day $14(110.2 \pm 27.1 \mathrm{~mL} / 100 \mathrm{~g} / \mathrm{min}, p=0.044)$ and 30 (110.2 $\pm 20.8 \mathrm{~mL} / 100 \mathrm{~g} / \mathrm{min}, p=0.039)$ were significantly higher compared to day $1(79.7 \pm 27.6$ $\mathrm{mL} / 100 \mathrm{~g} / \mathrm{min}$ ) post ischemia (Sidak's post hoc test).

A significant difference between infarct and contralateral striatum was observed at day 7 ( $p=$ 0.042) post ischemia. No significant difference between both hemispheres was observed at days 21 and 30 post ischemia. 
In PLX5622-treated mice, mean CBF values in the contralateral side significantly increased from day $1(98.6 \pm 7.4 \mathrm{~mL} / 100 \mathrm{~g} / \mathrm{min})$ to days $7(137.7 \pm 4.08 \mathrm{~mL} / 100 \mathrm{~g} / \mathrm{min}, p=0.026)$ and $14(135.3 \pm$ $25.45 \mathrm{~mL} / 100 \mathrm{~g} / \mathrm{min}, p=0.044$ ), followed by a significant decrease at day 21 post ischemia. Mean CBF values at day $21(99.9 \pm 5.19 \mathrm{~mL} / 100 \mathrm{~g} / \mathrm{min})$ were significantly decreased compared to day 3 $(137.7 \pm 12.1 \mathrm{~mL} / 100 \mathrm{~g} / \mathrm{min}, p=0.030)$ and $7(p=0.028)$ post ischemia (Sidak's post hoc test).

Mean CBF values within the infarct were increased at day $3(131.4 \pm 20.8 \mathrm{~mL} / 100 \mathrm{~g} / \mathrm{min}, p=0.047)$, $7(118.6 \pm 9.6 \mathrm{~mL} / 100 \mathrm{~g} / \mathrm{min}, p=0.01)$ and $14(129.1 \pm 26.0 \mathrm{~mL} / 100 \mathrm{~g} / \mathrm{min}, p=0.037)$ compared to day $1(80.0 \pm 10.2 \mathrm{~mL} / 100 \mathrm{~g} / \mathrm{min})$ post ischemia (Sidak's post hoc). Mean CBF values within the infarct at day $21(89.1 \pm 10.4 \mathrm{~mL} / 100 \mathrm{~g} / \mathrm{min})$ were decreased compared to day $7(p=0.02)$ post ischemia.

In PLX5622-treated mice, mean CBF within the infarct was significantly lower than in the contralateral side at day $1(p=0.034), 7(p=0.012)$ and $21(p=0.046)$ post.

Data are depicted as mean $\pm \mathrm{SD}\left(\mathrm{n}=8 /\right.$ group) $\left({ }^{*} p<0.05,{ }^{* *} p<0.01,{ }^{* * *} p<0.005,{ }^{*}\right.$ vs day $1, \#$ vs treatment, + vs contralateral, $\$$ vs day 21$)$. 
A Control
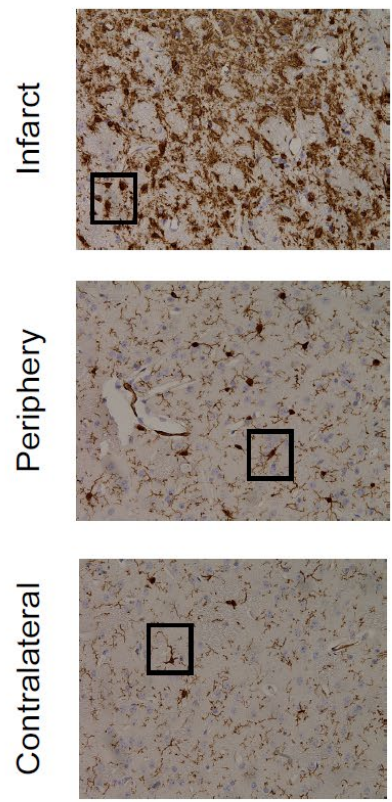

PLX5622
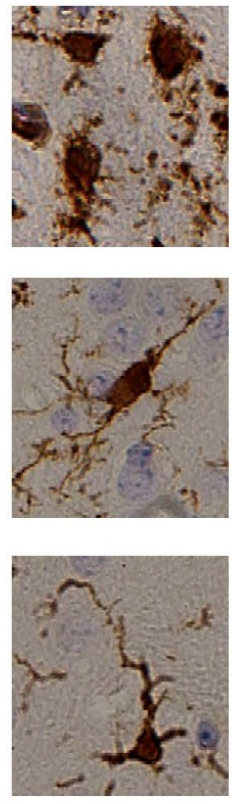
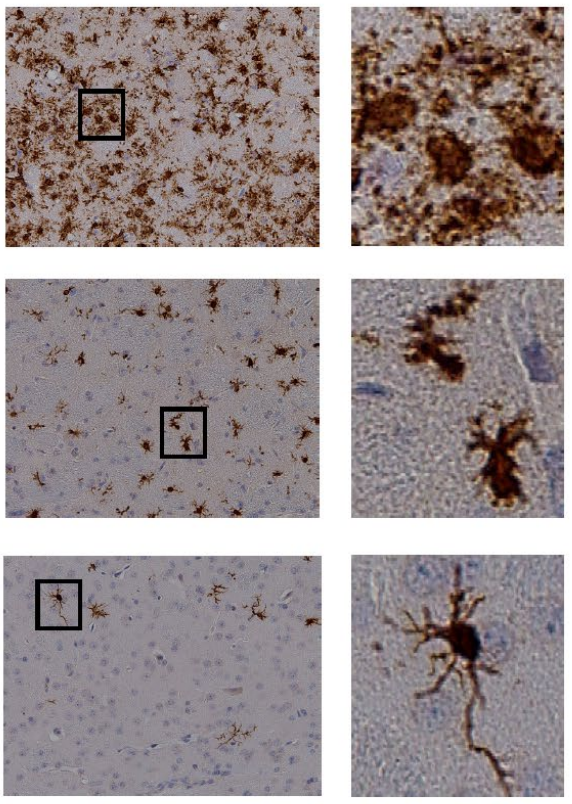

B

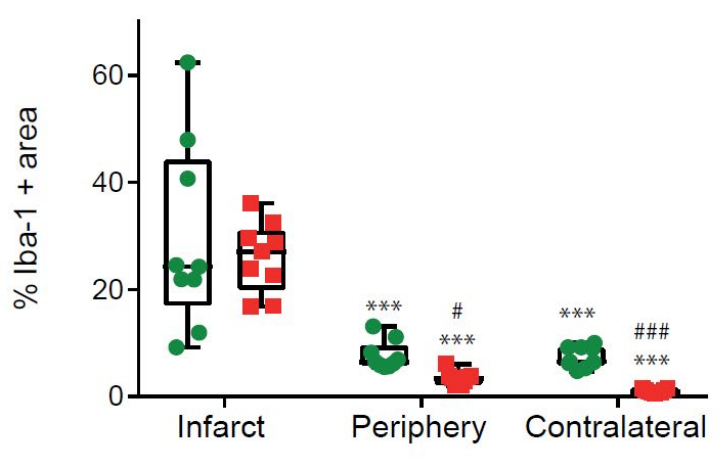

- Control

- PLX5622

\section{Supplementary Figure 9. PLX5622 treatment modulated Iba-1 immunoreactivity in PLX5622-}

treated mice brains at day 35 post ischemia. (A) Representative images of Iba-1 immunoreactivity within the infarct, at the periphery of the infarct and contralateral side in control and PLX5622-treated mice at day 35 post ischemia. PLX5622 treatment may modulate microglia reactivity, as indicated by the difference in morphology. At the periphery of the infarct, microglial cells have elongated thin processes in control mice while they showed shorter thicker processes in PLX5622 treated mice. (B) Quantification of Iba-1 immunoreactivity within the 
infarct, at the periphery of the infarct and contralateral side expressed in percentage of stained area. Therapy effect was observed at the periphery and contralateral striatum, where PLX5622treated mice showed a lower percentage of $\mathrm{Iba}-1^{+}$area $(n=3 / g r o u p, 3$ fields of view per mouse and region).

Data were analysed by two-way RM ANOVA followed by Sidak's post hoc test for multiple comparisons $\left({ }^{*} p<0.05,{ }^{* *} p<0.01,{ }^{* * *} p<0.005 ;{ }^{*}\right.$ vs infarct, \# vs treatment). 
A
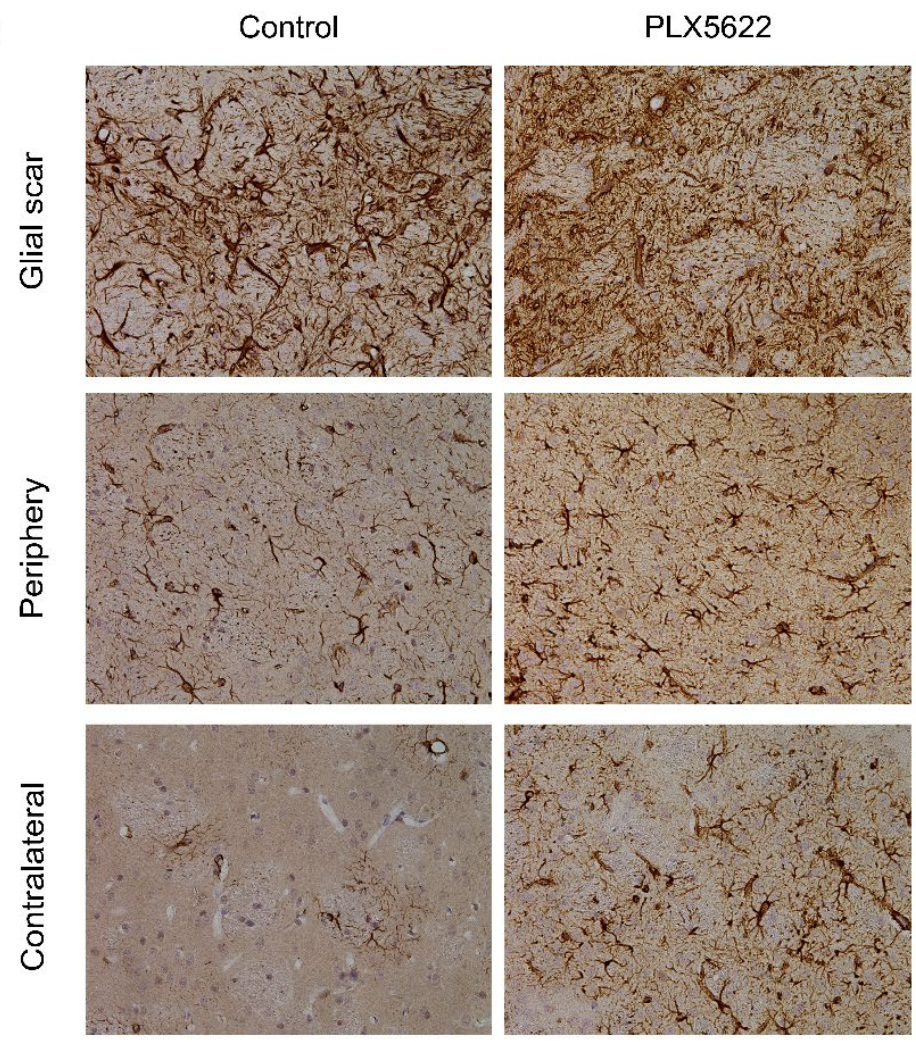

B

- Control

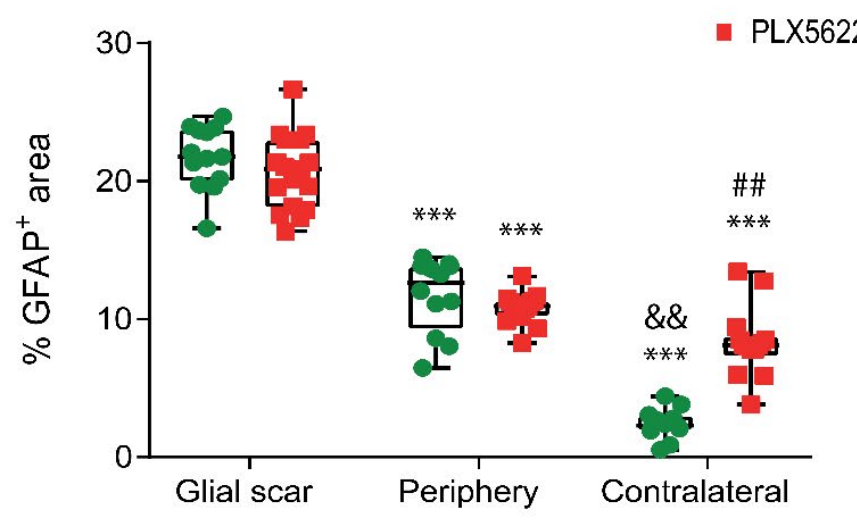

Supplementary Figure 10. PLX5622 treatment increased the number of GFAP ${ }^{+}$cells in the contralateral side after long-term CSF-1R inhibition. (A) Representative GFAP staining in three regions (glial scar, periphery and contralateral) for both experimental groups. (B) A treatment effect was observed in the contralateral side: PLX5622-treated mice showed a higher percentage of $\mathrm{GFAP}^{+}$area compared to control mice $(p=0.006)$. The percentage of $\mathrm{GFAP}^{+}$area at the glial 
scar and periphery was unaffected by PLX5622 treatment. (Control: $n=4$, PLX5622: $n=5,3$ fields of view per region per mouse).

Data were analysed by two-way RM ANOVA followed by Sidak's post hoc test for multiple comparisons $(* * p<0.01, * * * p<0.005 ; *$ vs glial scar, \& vs periphery, \# vs treatment). 


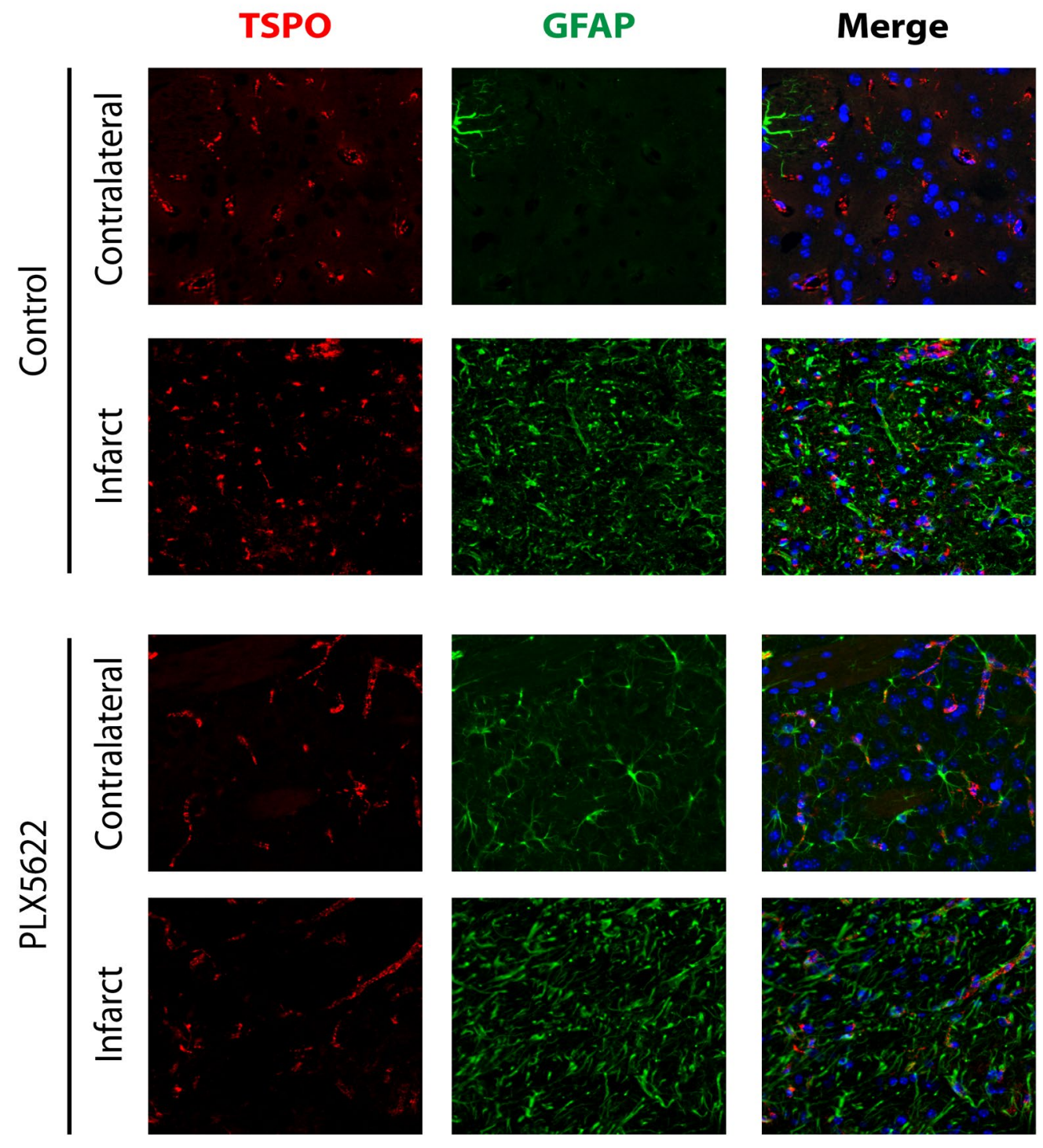

Supplementary Figure 11. GFAP-positive cells are mostly TSPO-negative at day 35 post ischemia. Representative co-staining of TSPO (red) and GFAP (green) within the infarct and contralateral side and the corresponding merged image for both experimental groups. No or few $\mathrm{GFAP}^{+}$cells were co-localizing with TSPO within the infarct and contralateral striatum for both experimental group. PLX5622 did not affect the percentage of GFAP ${ }^{+}$area within the infarct but increased GFAP expression in the contralateral side of PLX5622-treated mice compared to control. 
A

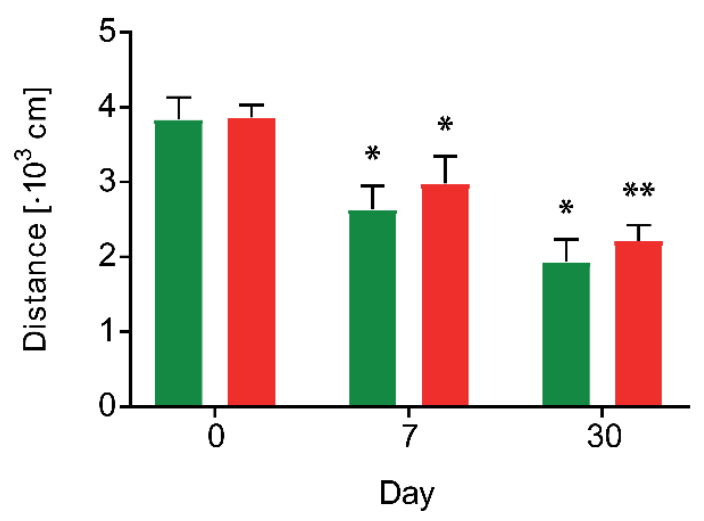

- Control
B

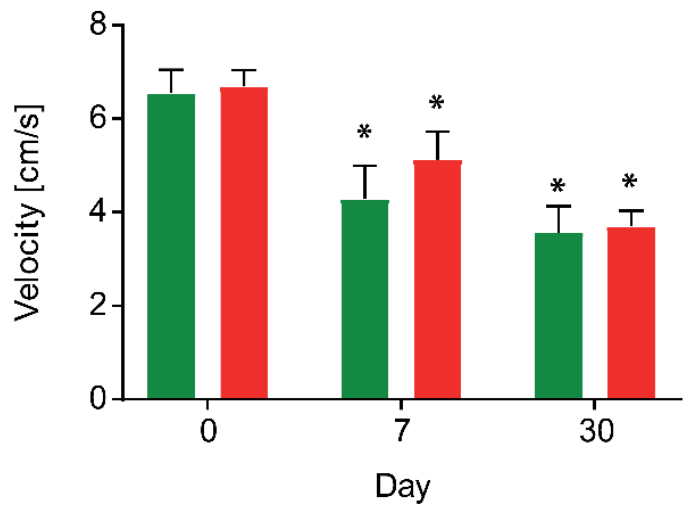

DLX5622

Supplementary Figure 12. Long-term PLX5622 treatment did not improve general locomotion.

(A) Travelled distance and (B) velocity were assessed as indexes of global locomotion using the open field test. Overall, no treatment effect was observed between both experimental groups. Walking distance $\left(R^{2}=0.25\right)$ and velocity $\left(R^{2}=0.30\right)$ did not correlate with 18F-DPA-714 tracer uptake within the infarct at day 14 post ischemia. Statistical analysis was carried out with twoway RM ANOVA followed by Holm Sidak's post hoc test for multiple comparisons ( $\mathrm{n}=8 /$ group). Data are expressed as mean $\pm \operatorname{SEM}\left({ }^{*} p<0.05,{ }^{*} p<0.01,{ }^{*}\right.$ vs day 1$)$. 


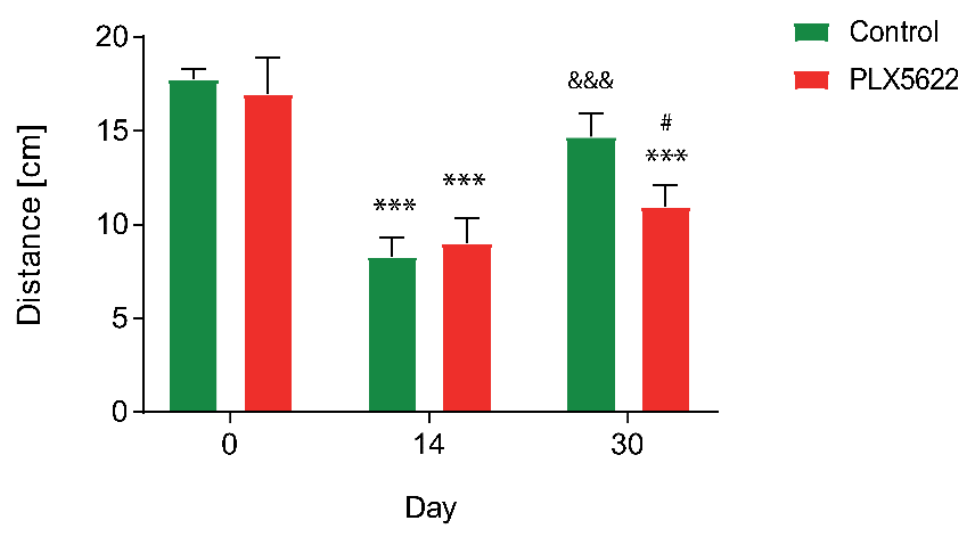

Supplementary Figure 13. Long-term PLX5622 treatment impaired coordination. PLX5622treated mice showed impaired coordination/balance compared to control mice at day 30 post ischemia. The run distance on the rotarod did not correlate with 18F-DPA-714 tracer uptake within the infarct at day 14 post ischemia $\left(R^{2}=0.11\right)$. Statistical analysis was carried out with twoway RM ANOVA s followed by Holm Sidak's post hoc test for multiple comparisons ( $n=8 /$ group). Data are expressed as mean $\pm \operatorname{SEM}\left({ }^{* * *} p<0.005 ; *\right.$ vs day $1, \&$ vs day $14, \#$ vs treatment). 


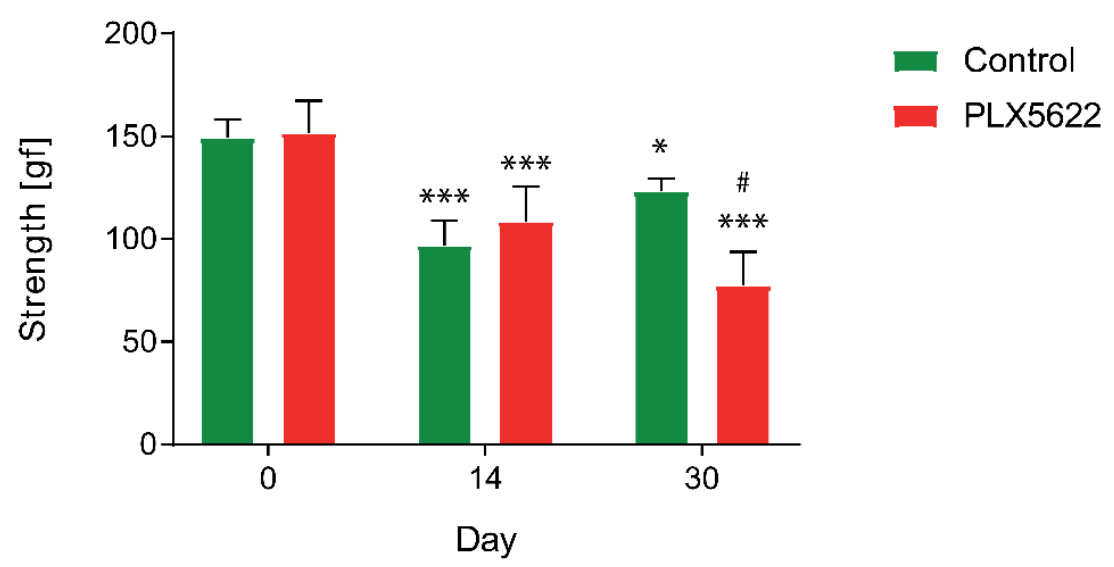

Supplementary Figure 14. Long-term PLX5622 treatment impaired forelimbs strength recovery after ischemia. Treatment effect on forelimbs strength was observed at day 30 post ischemia. PLX5622-treated mice showed less strength in the forelimbs compared to control mice. Forelimbs strength did not correlate with 18F-DPA-714 tracer uptake within the infarct at day 14 post ischemia $\left(R^{2}=0.21\right)$. Statistical analysis was carried out with two-way RM ANOVA followed by Holm Sidak's post hoc test for multiple comparisons ( $n=8 /$ group). Data are expressed as mean \pm $\operatorname{SEM}\left({ }^{*} p<0.05, * * * p<0.005 ; *\right.$ vs day $1, \&$ vs day $14, \#$ vs treatment). 


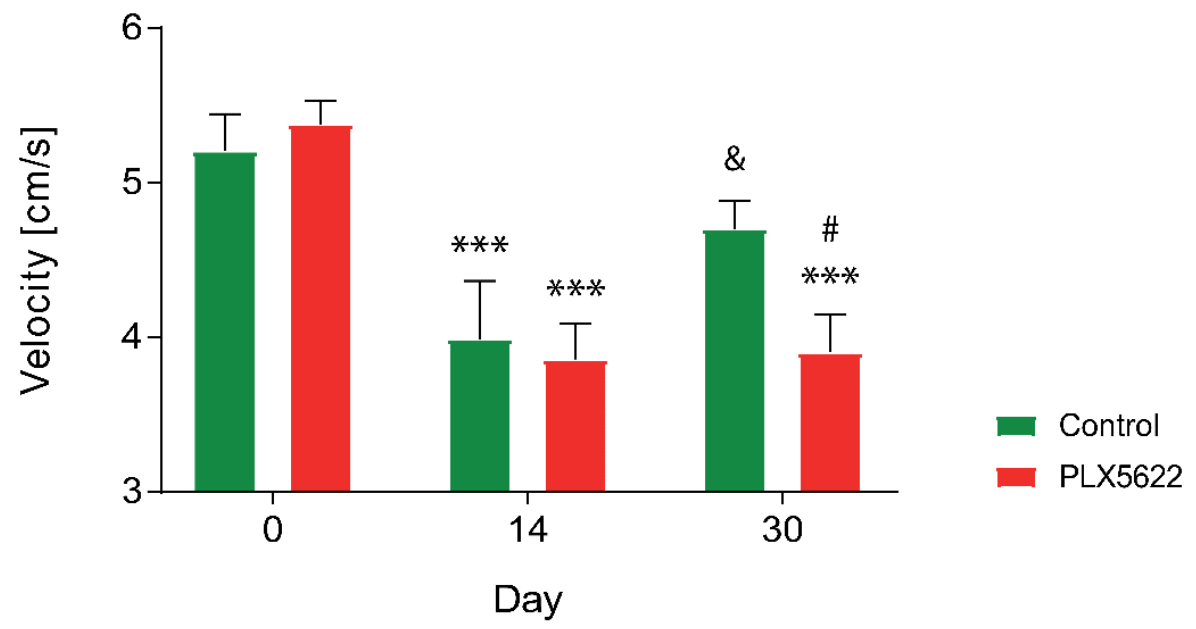

Supplementary Figure 15. PLX5622 treatment impaired long-term recovery of general motor functions. A treatment effect was observed at day 30 post ischemia: PLX5622-treated mice walked slower than control mice over the pole, indicative of impaired motor recovery. Velocity did not correlate with 18F-DPA-714 tracer uptake within the infarct at day 14 post ischemia $\left(R^{2}=\right.$ 0.34). Statistical analysis was carried out with two-way RM ANOVA followed by Holm Sidak's post hoc test for multiple comparisons ( $n=8 /$ group). Data are expressed as mean $\pm \operatorname{SEM}\left({ }^{* * *} p<0.005\right.$; * vs day $1, \&$ vs day $14, \#$ vs treatment). 